\title{
Tectonic significance of Triassic mafic rocks in the June Complex, Sanandaj-Sirjan zone, Iran
}

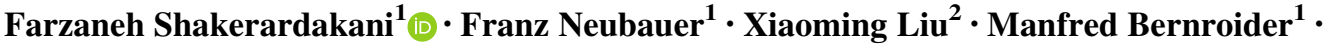 \\ Behzad Monfaredi ${ }^{3} \cdot{\text { Albrecht von } \text { Quadt }^{4}}^{4}$
}

Received: 13 December 2016/Accepted: 3 August 2017/Published online: 20 September 2017

(C) The Author(s) 2017. This article is an open access publication

\begin{abstract}
This study concentrates on the petrological and geochemical investigation of mafic rocks embedded within the voluminous Triassic June Complex of the central Sanandaj-Sirjan zone (Iran), which are crucial to reconstruct the geodynamics of the Neotethyan passive margin. The Triassic mafic rocks are alkaline to sub-alkaline basalts, containing 43.36-49.09 wt\% $\mathrm{SiO}_{2}, 5.19-20.61 \mathrm{wt} \% \mathrm{MgO}$ and $0.66-4.59 \mathrm{wt} \%$ total alkalis. Based on $\mathrm{MgO}$ concentrations, the mafic rocks fall into two groups: cumulates $(\mathrm{Mg} \#=51.61-58.94)$ and isotropic basaltic liquids $(\mathrm{Mg} \#=24.54-42.66)$. In all samples, the chondrite-normalized REE patterns show enrichment of light REEs with variable $(\mathrm{La} / \mathrm{Yb})_{\mathrm{N}}$ ratios ranging from 2.48 to 9.00 , which confirm their amalgamated OIB-like and E-MORB-like signatures. Enrichment in large-ion lithophile elements and depletion in high field strength elements (HFSE) relative to the primitive mantle further support this interpretation. No samples point to crustal contamination, all having undergone fractionation of olivine + clinopyroxene + plagioclase. Nevertheless, elemental data suggest that the substantial variations in $(\mathrm{La} / \mathrm{Sm})_{\mathrm{PM}}$ and $\mathrm{Zr} / \mathrm{Nb}$ ratios can be explained
\end{abstract}

Editorial handling: E. Gnos.

Farzaneh Shakerardakani

farzaneh.shakerardakani@sbg.ac.at

1 Department Geography and Geology, University of Salzburg, Hellbrunner Str. 34, 5020 Salzburg, Austria

2 State Key Laboratory of Continental Dynamics, Department of Geology, Northwest University, Northern Taibai Str. 229, Xi' an 710069, China

3 Institute of Earth Sciences, University of Graz, Universitätsplatz 2, $8010 \mathrm{Graz}$, Austria

4 Institute of Isotope Geology and Mineral Resources, ETH Zürich, 8092 Zurich, Switzerland by variable degrees of partial melting rather than fractional crystallization from a common parental magma. The high $(\mathrm{Nb} / \mathrm{Yb})_{\mathrm{PM}}$ ratio in the alkaline mafic rocks points to the mixing of magmas from enriched and depleted mantle sources. Abundant OIB alkaline basalts and rare E-MORB appear to be linked to the drifting stage on the northern passive margin of the Neotethys Ocean.

Keywords Sanandaj-Sirjan zone $\cdot$ June Complex $\cdot$ Mafic rocks $\cdot$ Geochemistry $\cdot$ Neotethys

\section{Introduction}

Late Palaeozoic (Permian) rifting of the future Neotethys Ocean has formed ribbon continental fragments in Iran that broke away from the northern margin of Gondwana (e.g., Berberian and King 1981; Şengör 1990; Stampfli et al. 1991; Stampfli and Borel 2002; Mohajjel et al. 2003; Ghasemi and Talbot 2006; Agard et al. 2011; Richards 2015) although relationships to Triassic terrane accretion to the north of Central Iran remains partly unclear (Buchs et al. 2013; Zanchi et al. 2015). This contribution deals with the Sanandaj-Sirjan zone (SSZ; Fig. 1), which represents the passive margin facing the Neotethyan Ocean in the south and was part of the Cimmerian microcontinent during Triassic times (Alavi 1994; Agard et al. 2011; Hassanzadeh and Wernicke 2016 and references therein).

Late Carboniferous-Permian rifting of the Neotethys Ocean was followed by a phase of pronounced subsidence and shallow marine and basinal sedimentation in Permian through Triassic time (e.g., Stampfli et al. 1991, 2001; Besse et al. 1998; Mohajjel and Fergusson 2014; Hassanzadeh and Wernicke 2016). Hassanzadeh and Wernicke (2016) recently considered the SSZ as an archetype of 


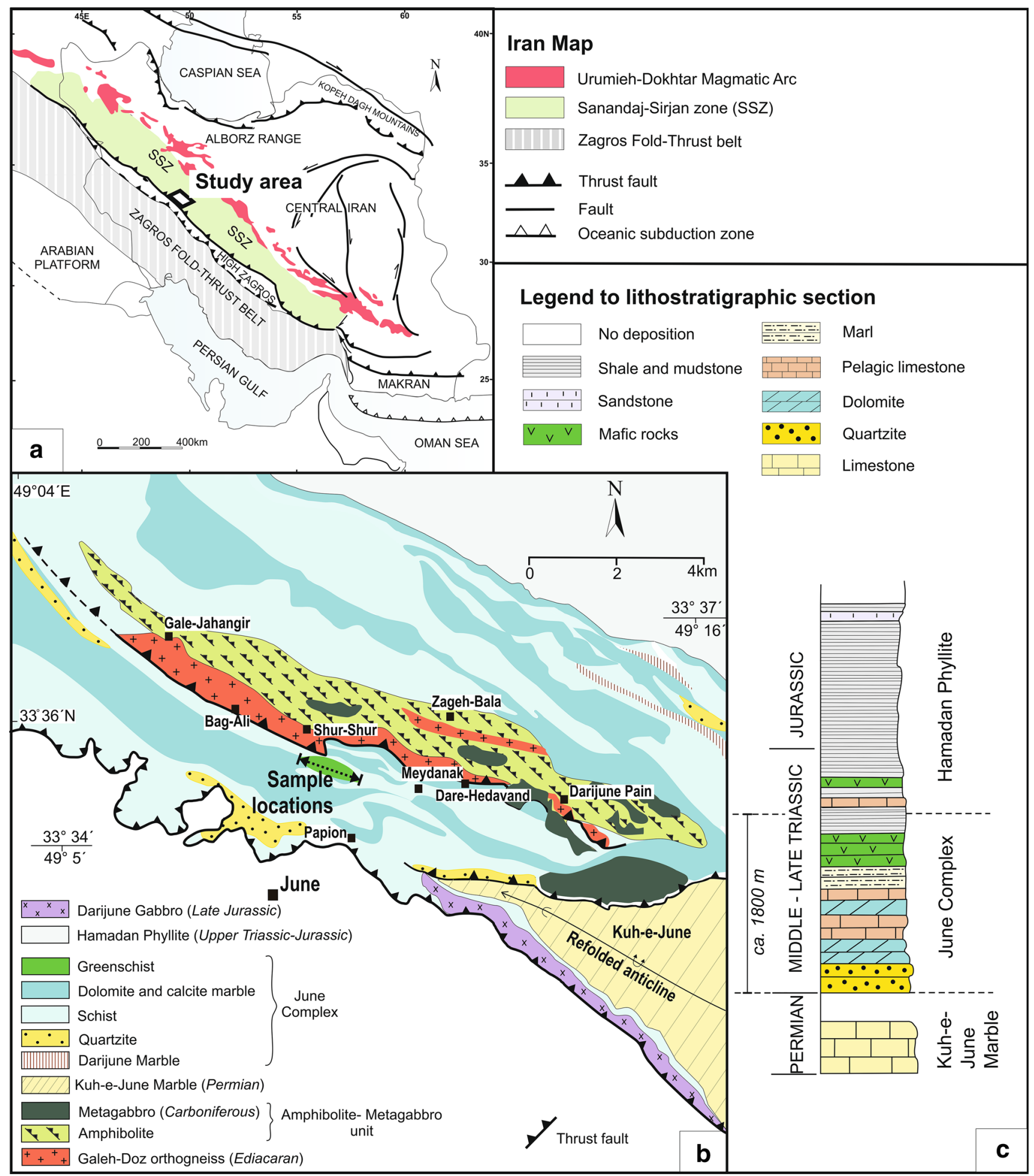

Fig. 1 a Tectonic zones of Iran after Alavi (1994). b Simplified geological map of the Dorud-Azna region (after Mohajjel and Fergusson 2000). c Simplified lithostratigraphic section of Triassic and Jurassic sedimentary units of the Dorud-Azna region (after Mohajjel et al. 2003)

passive continental margin subsequently, during Jurassic, transformed into an active continental margin.

A remarkable example of low-grade metamorphic sedimentary units recording the Carboniferous rifting, Permian shallow water deposition and Triassic to Jurassic basinal sedimentation on a passive continental margin is exposed in the Dorud-Azna region, Western Iran, in the central part of SSZ (Fig. 1a, b). Structural studies comprise that these 
Table 1 Locations and magmatic and metamorphic mineralogy of investigated samples used in this paper

\begin{tabular}{|c|c|c|c|c|c|}
\hline $\begin{array}{l}\text { Sample } \\
\text { no. }\end{array}$ & $\begin{array}{l}\text { Latitude } \\
(\mathrm{N})\end{array}$ & $\begin{array}{l}\text { Longitude } \\
\text { (E) }\end{array}$ & Rock type & Magmatic mineral assemblage & Metamorphic mineral assemblage \\
\hline P-105 & $33^{\circ} 34^{\prime} 59^{\prime \prime}$ & $49^{\circ} 09^{\prime} 37^{\prime \prime}$ & $\begin{array}{l}\text { Talc-bearing } \\
\text { greenschist }\end{array}$ & $\begin{array}{l}\text { Clinopyroxene, amphibole, } \\
\text { plagioclase, K-feldspar, apatite }\end{array}$ & $\begin{array}{l}\text { Plagioclase, talc, epidote, clinochlore, } \\
\text { rutile }\end{array}$ \\
\hline P-149 & $33^{\circ} 35^{\prime} 05^{\prime \prime}$ & $49^{\circ} 09^{\prime} 30^{\prime \prime}$ & $\begin{array}{l}\text { Talc-bearing } \\
\text { greenschist }\end{array}$ & $\begin{array}{l}\text { Clinopyroxene, plagioclase, } \\
\text { K-feldspar, apatite, ilmenite }\end{array}$ & $\begin{array}{l}\text { Actinolite, plagioclase, talc, chlorite, rutile, } \\
\text { ilmenite }\end{array}$ \\
\hline $\mathrm{P}-147$ & $33^{\circ} 35^{\prime} 03^{\prime \prime}$ & $49^{\circ} 09^{\prime} 13^{\prime \prime}$ & $\begin{array}{l}\text { Actinolite-bearing } \\
\text { greenschist }\end{array}$ & Amphibole, clinopyroxene, apatite & Amphibole, chlorite, epidote, rutile \\
\hline P-154 & $33^{\circ} 34^{\prime} 59^{\prime \prime}$ & $49^{\circ} 09^{\prime} 37^{\prime \prime}$ & $\begin{array}{l}\text { Actinolite-bearing } \\
\text { greenschist }\end{array}$ & Amphibole, clinopyroxene, apatite & Amphibole, chlorite, epidote, rutile \\
\hline $\mathrm{J}-212$ & $33^{\circ} 35^{\prime} 40^{\prime \prime}$ & $49^{\circ} 09^{\prime} 12^{\prime \prime}$ & $\begin{array}{l}\text { Coarse-grained } \\
\text { metagabbroic } \\
\text { greenschist }\end{array}$ & $\begin{array}{l}\text { Amphibole, plagioclase, } \mathrm{K} \text {-feldspar, } \\
\text { quartz, apatite, opaque minerals }\end{array}$ & $\begin{array}{l}\text { Amphibole, plagioclase, quartz, chlorite, } \\
\text { epidote, sphene, rutile, opaque minerals }\end{array}$ \\
\hline $\mathrm{P}-124$ & $33^{\circ} 35^{\prime} 06^{\prime \prime}$ & $49^{\circ} 09^{\prime} 02^{\prime \prime}$ & $\begin{array}{l}\text { Fine-grained } \\
\text { metagabbroic } \\
\text { greenschist }\end{array}$ & $\begin{array}{l}\text { Amphibole, plagioclase, K-feldspar, } \\
\text { apatite, opaque minerals }\end{array}$ & $\begin{array}{l}\text { Amphibole, plagioclase, quartz, chlorite, } \\
\text { epidote, sphene, rutile, opaque minerals }\end{array}$ \\
\hline P-136 & $33^{\circ} 35^{\prime} 46^{\prime \prime}$ & $49^{\circ} 09^{\prime} 10^{\prime \prime}$ & $\begin{array}{c}\text { Medium-grained } \\
\text { metagabbroic } \\
\text { greenschist }\end{array}$ & $\begin{array}{l}\text { Amphibole, plagioclase, K-feldspar, } \\
\text { apatite, opaque minerals }\end{array}$ & $\begin{array}{l}\text { Amphibole, plagioclase, quartz, chlorite, } \\
\text { epidote, sphene, rutile, opaque minerals }\end{array}$ \\
\hline P-119 & $33^{\circ} 35^{\prime} 44^{\prime \prime}$ & $49^{\circ} 09^{\prime} 09^{\prime \prime}$ & $\begin{array}{l}\text { Fine-grained } \\
\text { metagabbroic } \\
\text { greenschist }\end{array}$ & $\begin{array}{l}\text { Amphibole, plagioclase, K-feldspar, } \\
\text { apatite, opaque minerals }\end{array}$ & $\begin{array}{l}\text { Amphibole, plagioclase, quartz, chlorite, } \\
\text { epidote, sphene, rutile, opaque minerals }\end{array}$ \\
\hline $\mathrm{P}-125$ & $33^{\circ} 35^{\prime} 06^{\prime \prime}$ & $49^{\circ} 09^{\prime} 21^{\prime \prime}$ & $\begin{array}{l}\text { Fine-grained } \\
\text { metagabbroic } \\
\text { greenschist }\end{array}$ & $\begin{array}{l}\text { Amphibole, plagioclase, K-feldspar, } \\
\text { apatite, opaque minerals }\end{array}$ & $\begin{array}{l}\text { Amphibole, plagioclase, quartz, chlorite, } \\
\text { epidote, sphene, rutile, opaque minerals }\end{array}$ \\
\hline P-134 & $33^{\circ} 35^{\prime} 53^{\prime \prime}$ & $49^{\circ} 09^{\prime} 10^{\prime \prime}$ & $\begin{array}{l}\text { Medium-grained } \\
\text { metagabbroic } \\
\text { greenschist }\end{array}$ & $\begin{array}{l}\text { Amphibole, plagioclase, K-feldspar, } \\
\text { apatite, opaque minerals }\end{array}$ & $\begin{array}{l}\text { Amphibole, plagioclase, quartz, chlorite, } \\
\text { epidote, sphene, rutile, opaque minerals }\end{array}$ \\
\hline
\end{tabular}

metasedimentary units are imbricated with older, highergrade metamorphic units. These are from footwall to hangingwall: (1) Permian Marble and overlying Triassic Complex metamorphosed within greenschist facies conditions and, to the southeast, intruded by the Jurassic Darijune gabbro, tectonically overlain by (2) amphibolite-facies Panafrican orthogneiss and (3) Carboniferous Amphibolite-Metagabbro unit (Mohajjel et al. 2003; Shakerardakani et al. 2015; Fergusson et al. 2016).

This contribution aims to study the mafic rocks within the Triassic unit (1) with focus on their geochemical characteristics and tectonic environment. The new data gives insights into the nature of magmatism in the passive margin of Neo-Tethys.

\section{Geological setting}

The Dorud-Azna region is situated in the central part of the SSZ (Fig. 1b). The SSZ is a polyphase metamorphic terrain with a metamorphic Panafrican and Carboniferous basement overlain by Permian to Upper Cretaceous sedimentary successions, which experienced metamorphism ranging from greenschist to eclogite facies conditions (e.g.,
Agard et al. 2005; Davoudian et al. 2011, 2016; Shakerardakani et al. 2015; Hassanzadeh and Wernicke 2016). Agard et al. (2011) suggest that the metamorphosed Permian and Triassic rocks are thrust along the Main Zagros thrust (MZT) on top of the Crush Zone (Gidon et al. 1974).

The studied Triassic Complex is a mixed low-grade metamorphic sequence of metasedimentary and subordinate mafic rocks (Fig. 1c), which is tectonically overlain by a Panafrican basement with the Gondwanan affiliation of orthogneiss and various in part Carboniferous-aged amphibolites and metagabbros (Nutman et al. 2014; Shakerardakani et al. 2015, 2017; Fergusson et al. 2016).

The Triassic metasediments consists of three members including a quartzite-dominated base, a calcite marble at the middle level, and an upper level with schists interbedded mafic rocks (Mohajjel and Fergusson 2000; Fig. 1b, c). A Middle to Late Triassic age is defined by fossils in dolomite and calcite marbles (Sahandi et al. 2006) and Permian fossils in the underlying dolomite marbles (Sahandi et al. 2006).

Interlayered mafic rocks consist of various types of epidote-amphibolite and greenschists, which have experienced upper greenschist to epidote-amphibolite facies metamorphism (Shakerardakani et al. 2015). 

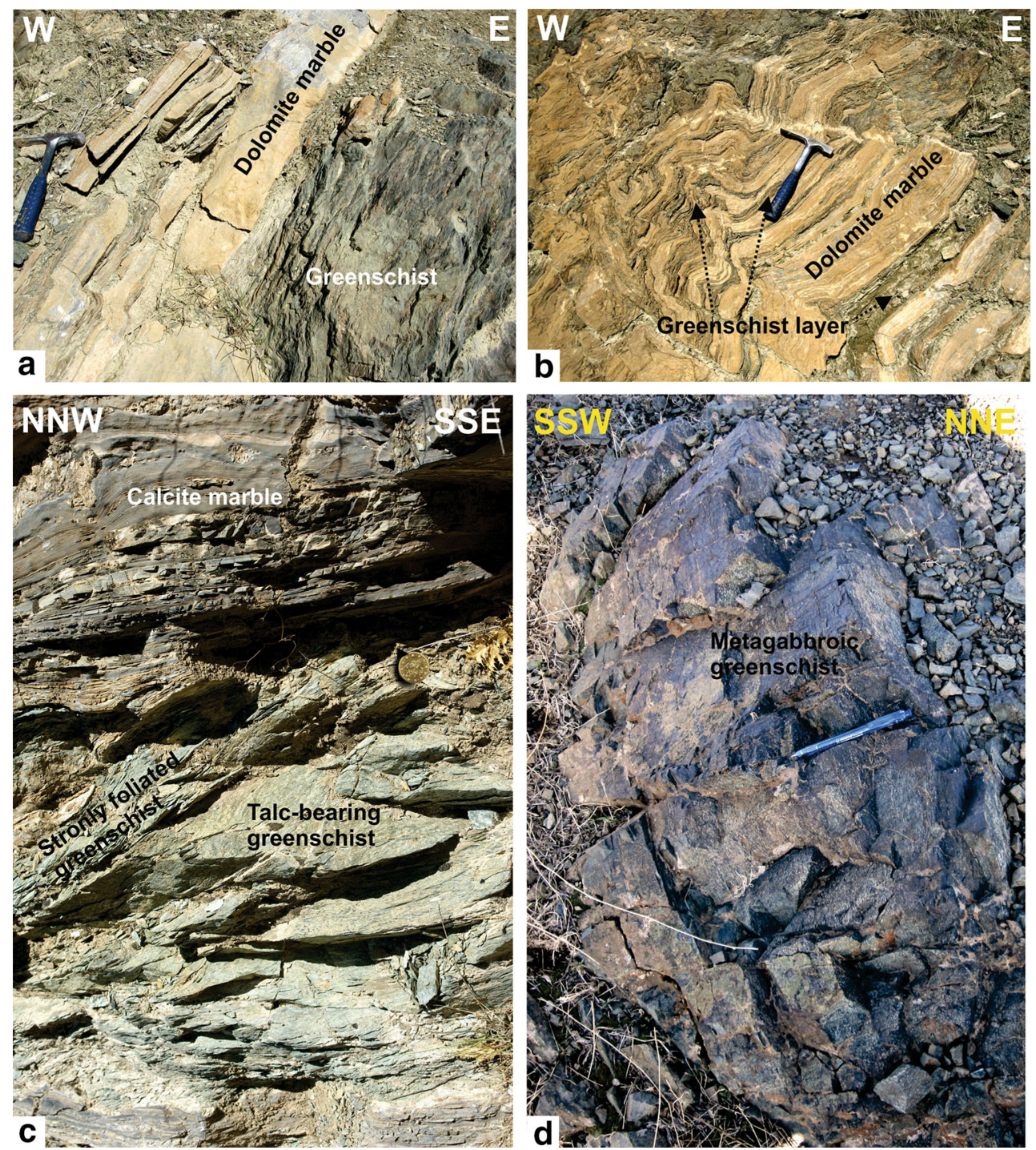

Fig. 2 Field photographs of mafic and other rocks from the study area. a Mafic rocks close to the contact to the metasedimentary rocks containing metasandstone layers. b Folded dolomite marble interlayered with greenschists. c Representative lensoid talc-bearing

The Middle-Upper Triassic succession of the study area is tectonically bordered by the Panafrican orthogneiss and Upper Triassic-Jurassic phyllites to the north and is, to the south structurally overlying unmetamorphic Upper Jurassic to Lower Cretaceous andesitic lavas and pyroclastic rocks (Fig. 1b).

\section{Field relation and petrography}

The samples used for this study were taken to the southeast of the Shur-Shur village (Fig. 1b; Table 1), where they form boudinaged lenses or are interlayered within marbles are aligned for a distance over more than $1 \mathrm{~km}$. The contact

greenschist within quartzite/metachert layer. Note decreasing intensity of foliation away from contact to metasediment. d Strongly sheared and boudinaged fine-grained metagabbroic greenschist

to host rocks is sharp without evidence of either intense shearing or contact metamorphism.

Petrographically, the mafic rocks can be categorized as actinolite-bearing greenschist (Fig. 2a, b), talc-bearing greenschist and metagabbroic greenschists (epidote-amphibolite). Talc- and actinolite-bearing greenschists mainly crop out in form of lens-shaped boudins in dolomite and calcite marble (Fig. 2c, d). The metagabbroic greenschists (epidote-amphibolites) form kilometer-scaled layers intercalated with calcareous phyllites and impure marbles (Fig. 2d).

Metagabbroic greenschists (epidote-amphibolite) vary from medium- to fine-grained, although some exposures are coarse-grained in places (Fig. 2d). The observed 
Fig. 3 Photomicrographs (a-c, e, f) and back-scattered electron (BSE) image (d) of various metagabbroic greenschists (epidote-amphibolite), and talcand actinolite-bearing greenschists. a Foliated, finegrained metagabbroic greenschist (epidoteamphibolite). b Talc-bearing greenschist showing euhedral clinopyroxene porphyroclast surrounded by talc and chlorite ribbons. c, d Clinopyroxene phenocryst intergrown with brownish hornblende.

e Actinolite porphyroclast in actinolite-bearing greenschist. Mineral abbreviations: $C p x$ clinopyroxene, Amp amphibole, $\mathrm{Ep}$ epidote, Act actinolite, $\mathrm{Hbl}$ hornblende, $\mathrm{Pl}$ plagioclase, $\mathrm{Ilm}$ Ilmenite, $(\mathrm{Fe}) \mathrm{Mg}$ - $\mathrm{Hbl}$ Ferromagnesio-hornblende, $T l c$ talc, Chl chlorite
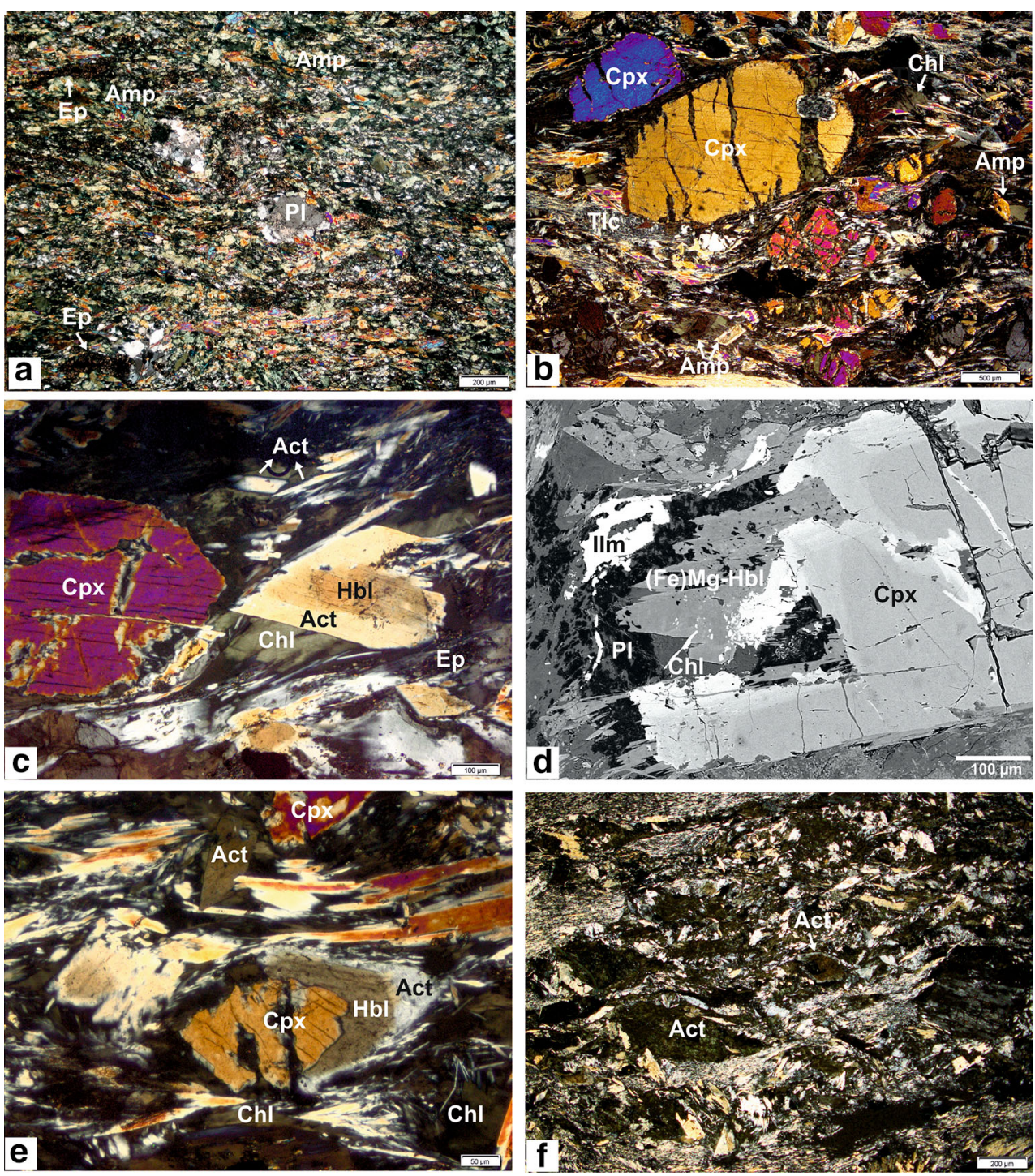

textures include porphyritic and ophitic ones. Magmatic minerals include plagioclase and amphibole (Table 1), which are in textural equilibrium. Along margins of these minerals and the matrix are overprinted by fine-grained metamorphic mineral assemblages including chlorite, epidote, quartz, and calcite, which formed within upper greenschist facies metamorphic conditions (Fig. 2c) implying some shear along the contact to host metasediments. The medium- to fine-grained metagabbroic greenschists are green to gray, porphyritic rocks, containing up to $10 \mathrm{vol} \%$ ca. $2-4 \mathrm{~mm}$ large phenocryst of magmatic amphibole, plagioclase, and rare K-feldspar and microcrystalline metamorphic aggregates of feldspar, quartz, epidote, titanite, chlorite, sericite and calcite (Fig. 3a; Table 1).

The talc-bearing greenschists are medium- to coarsegrained with the characteristic assemblage of clinopyroxene, amphibole, plagioclase and K-feldspar (Fig. 3b).
Large aggregates of metamorphic talc, chlorite and ilmenite have replaced large magmatic clinopyroxene (up to ca. $4 \mathrm{~mm}$ ), few amphibole phenocrysts or plagioclase crystals. Although the primary magmatic phases are commonly altered, the primary igneous texture is still well preserved. Clinopyroxene habits range from euhedral to anhedral and they present an oscillatory zoning, with locally overgrowth texture, or patchy zoning. The poikilitic magmatic clinopyroxene is occasionally rimmed by large, potentially magmatic brownish amphibole and fine-grained metamorphic actinolite (Fig. 3c, d). The amphibole grains reveal variable retrogression with irregular brownish patches in the interior of the grains surrounded by greenish amphibole, with actinolite representing the texturally latest amphibole forming the rims or occurring along cleavage planes together with chlorite. Late-magmatic brown amphibole forms poikilitic grains and overgrowths surrounding clinopyroxene, and dominates the intercumulate 


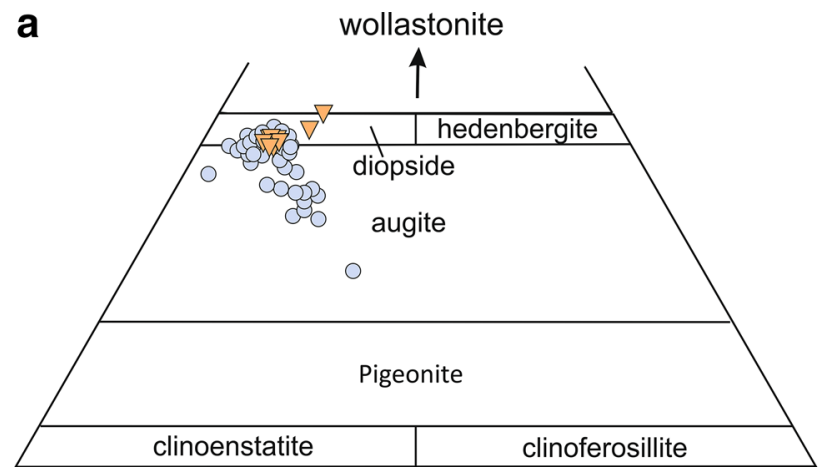

Enstatite

Ferrosilite
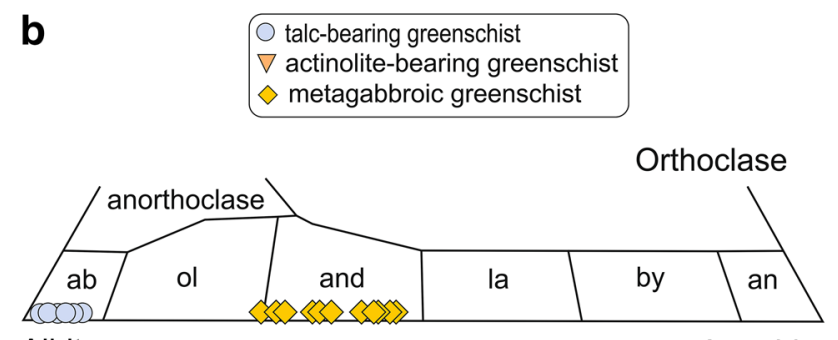

Albite

Anorthite

Fig. 4 a Pyroxene quadrilateral plot (Morimoto 1989) showing clinopyroxene compositions of talc- and actinolite bearing greenschist from studied mafic rocks. b Albite-anorthite-orthoclase diagram showing the compositions of plagioclase from talc-bearing and metagabbroic greenschists. $a b$ albite, ol oligoclase, and andesine, $l a$ labradorite, by bytownite, an anorthite

assemblage (Fig. 3e). Plagioclase has been part of the magmatic assemblage but is largely replaced by albite and dense mats of sericite and chlorite. K-feldspar forms tabular crystals of magmatic origin, and fine-grained recrystallized masses or irregular aggregates.

The dark greenish-gray actinolite-bearing greenschists are medium-grained and characterized by the mineral assemblage of magmatic amphibole, clinopyroxene, and the accessories like apatite and rutile. Euhedral to subhedral porphyroclastic amphibole grains form the dominant magmatic mineral in this rock as well as fine grains in the recrystallized matrix. Locally, some amphiboles occur as results of reactions of clinopyroxene. The amphibole porphyroclasts exhibit an optical color zoning ranging from yellow brownish magmatic cores to greenish metamorphic rims (Fig. 3f). However, the occurrence of magnesiohornblende, pargasite, and edenite (see below), locally with magmatic or metamorphic textures, indicates that they were probably formed at the late-magmatic to earlymetamorphic stages (Sappin et al. 2012). Pale green clinopyroxene forms heavily fractured grains with ragged margins, which commonly becomes an unstable magmatic mineral phase and underwent metamorphic replacement by greenish amphibole and chlorite along margins and cleavage planes.

\section{Analytical methods}

From a major number of samples, six of most unaltered samples, two of each of the three described types, were selected for geochemical analysis. On mostly magmatic minerals of three samples, the composition was investigated by microprobe. On one non-cumulatic sample, $\mathrm{Sr}-\mathrm{Nd}$ isotopic composition was investigated. Details on electron microprobe work, geochemical work and $\mathrm{Sr}-\mathrm{Nd}$ isotopic work are given in the Appendix.

\section{Mineral chemistry}

\subsection{Clinopyroxene}

The average chemical composition of clinopyroxene for talc- and actinolite-bearing greenschists is shown in the Wo-En-Fs diagram (Fig. 4a). The relictic clinopyroxenes in actinolite-bearing greenschist are generally diopside in composition. $\mathrm{Mg} \#$ varies from 73.7 to 87.6 from core to rim. Most clinopyroxene phenocrysts of the talc-bearing greenschist are chemically zoned with a diopside core and an augite rim. The core is higher in $\mathrm{Mg} \#(0.83-0.87)$ and $\mathrm{Si}$ but poorer in $\mathrm{Al}$ content than the rim (Fig. 5a, b). The compositional profile of a well preserved clinopyroxene crystal of sample $\mathrm{P} 105$ shows a rim composition with an $\mathrm{Al}$ content between 0.145 and 0.191 (a.p.f.u.) suddenly decreasing to 0.099 a.p.f.u. in the core (Fig. 5b; Table 2). Conversely, the $\mathrm{Na}$ contents progressively increases from rim to core (from 0.017 to 0.033 a.p.f.u.).

\subsection{Feldspar}

Plagioclase in the talc-bearing greenschist is nearly pure albite $\left(\mathrm{Ab}_{93-98}\right)$, while plagioclase in the metagabbroic greenschist is andesine $\left(\mathrm{Ab}_{62-71}\right)$ (Fig. $4 \mathrm{~b}$; Table 3$)$. The alkali feldspar is commonly orthoclase $\left(\mathrm{Or}_{96}\right)$ in talcbearing greenschist.

\subsection{Amphibole}

All investigated amphiboles are Ca-amphiboles (Table 3; classification of Leake et al. 1997). The amphiboles of the metagabbroic greenschist are magnesio-hornblende. They have $\mathrm{Mg \#}$ range from 0.71 to 0.74 and relatively high $\mathrm{TiO}_{2}$ and $\mathrm{Al}_{2} \mathrm{O}_{3}$ contents (0.36-0.54 and 9.12-11.67 wt\%, respectively). In the talc-bearing greenschist, amphiboles correspond to magnesio-hornblende, ferri-magnesio-hornblende, ferri-tremolite, and actinolite with $\mathrm{Mg} \#$ between 0.72 and 0.80 . Actinolite has low $\mathrm{TiO}_{2}$ and $\mathrm{Al}_{2} \mathrm{O}_{3}$ contents (0.01-0.08 and 1.35-3.79 wt\%, respectively). Brown 

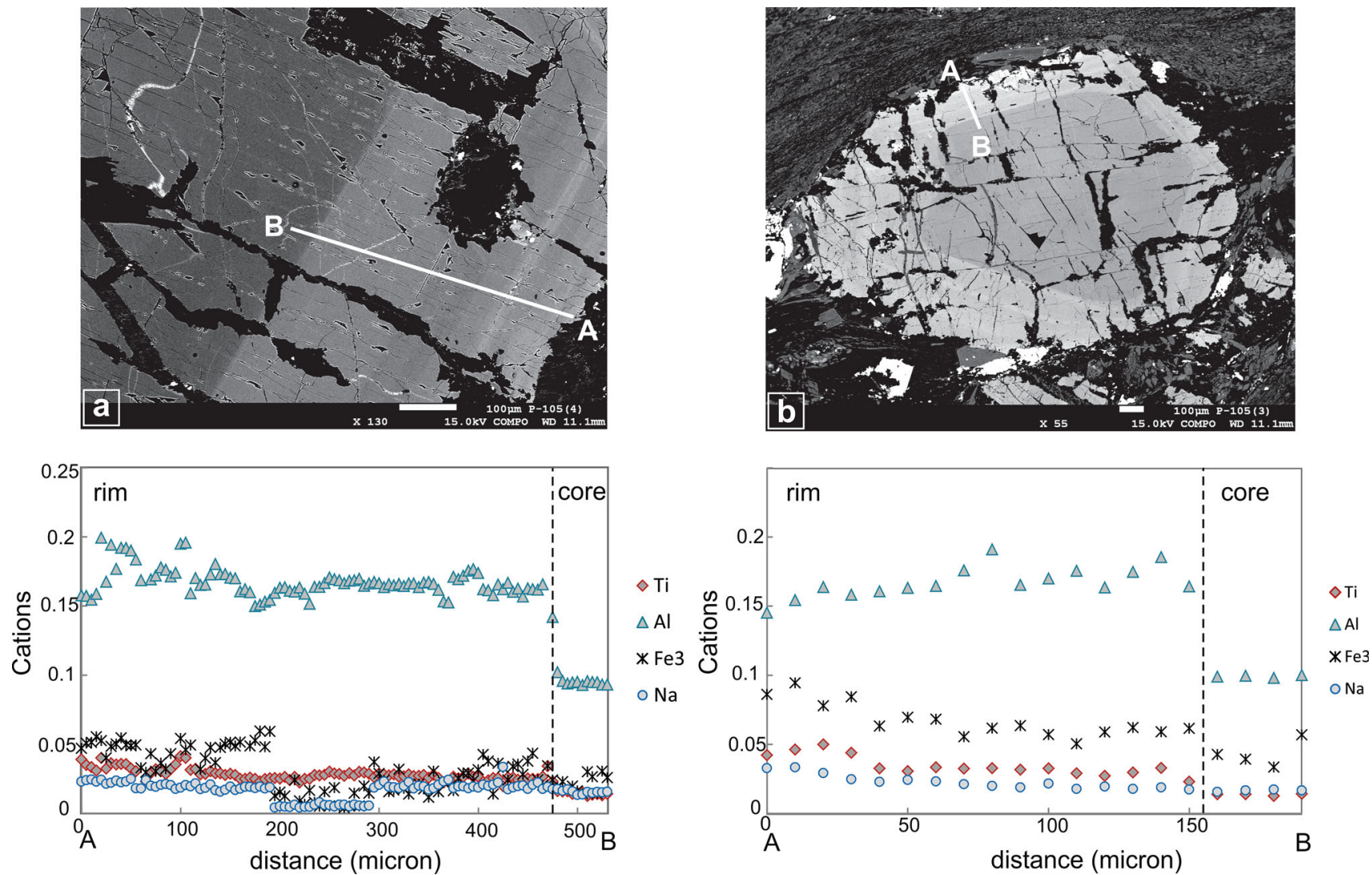

Fig. 5 a, b Rim to core chemical zoning (cations per 6 oxygens) in a clinopyroxene grain of talc-bearing greenschist from the June Complex

amphiboles at the contact to clinopyroxene are magnesiohornblende and ferri-magnesio-hornblende with $\mathrm{TiO}_{2}$ (0.46-1.06 wt\%) for the same $\mathrm{Mg \#}$ and $\mathrm{Al}_{2} \mathrm{O}_{3}$ contents of actinolites. In sheared actinolite-bearing greenschists, optically zoned amphibole porphyroclasts and matrix amphiboles occur. Some calcic amphiboles formed by decomposition of clinopyroxene by hydration processes. Porphyroclastic amphiboles show a relatively wide compositional range from magnesio-hornblende, ferro-actinolite, pargasite, edenite, to actinolite. All matrix amphiboles are actinolite. Its $\mathrm{Mg \#}$ varies between 0.67 and 0.85 . They have $\mathrm{TiO}_{2}$ and $\mathrm{Al}_{2} \mathrm{O}_{3}$ contents of $0.01-0.07$ and $0.19-1.20$ (wt\%) in actinolite to $0.75-3.39$ and 3.17-9.31 (wt\%) in magnesio-hornblende and edenite.

\section{Geochemistry}

Table 4 lists the whole-rock major and trace element data of six samples of talc- and actinolite-bearing greenschists, and metagabbroic greenschists (epidote-amphibolite). The new results (Table 4) are combined with four analyses from a preliminary, inconclusive survey (Shakerardakani et al. 2015). These included a cumulate talc-bearing greenschist and three talc-bearing and metagabbroic greenschists but no actinolite-bearing greenschists, which is suspected to represent the closest composition to basaltic liquids.

The immobile nature of $\mathrm{Ti}, \mathrm{Zr}, \mathrm{P}, \mathrm{Y}, \mathrm{Nb}$ means that they are neither affected by low-temperature alteration nor by metamorphism (e.g., Winchester and Floyd 1976; Pearce and Norry 1979) in the metamorphic P-T range experienced by the studied samples. This is supported by the fairly good correlation on variation diagrams of $\mathrm{Zr}$. vs. selected major elements (e.g., $\mathrm{SiO}_{2}, \mathrm{Al}_{2} \mathrm{O}_{3}, \mathrm{Fe}_{2} \mathrm{O}_{3}$, and $\mathrm{CaO}$ ), as well as incompatible trace elements (e.g., $\mathrm{Ti}, \mathrm{Nb}$, $\mathrm{Y}$ and Th), light (l) and heavy (h) rare earth elements (REE) (see below). Therefore, the analyses can be considered to represent primary geochemical features of the studied mafic rocks (e.g., Polat and Hofmann 2003; Saccani et al. 2010).

\subsection{Major element oxides}

$\mathrm{SiO}_{2}$ contents of the actinolite- and talc-bearing greenschists, and metagabbroic greenschists confirm that they are dominantly mafic $\left(\mathrm{SiO}_{2}=43.36-49.09 \mathrm{wt} \%\right.$ ). Alkali elements range from 0.20 to $4.29 \mathrm{wt} \%$ for $\mathrm{Na}_{2} \mathrm{O}$ and from 0.04 to $1.22 \mathrm{wt} \%$ for $\mathrm{K}_{2} \mathrm{O}$. They have relatively high $\mathrm{Al}_{2} \mathrm{O}_{3}$ (6.76-16.2 wt\%) and $\mathrm{Fe}_{2} \mathrm{O}_{3}$ (up to $13.76 \mathrm{wt} \%$ ) contents, 
Table 2 Representative analyses of clinopyroxene from the June Complex

\begin{tabular}{|c|c|c|c|c|c|c|c|c|c|c|}
\hline \multirow{3}{*}{$\begin{array}{l}\text { Sample-unit } \\
\text { Rock-type } \\
\text { Location }\end{array}$} & \multicolumn{10}{|c|}{ June Complex } \\
\hline & \multicolumn{10}{|c|}{ Talc-bearing greenschist } \\
\hline & \multicolumn{10}{|c|}{ Clinopyroxene-rim } \\
\hline $\mathrm{SiO}_{2}$ & 49.82 & 49.60 & 49.27 & 49.70 & 50.02 & 50.07 & 49.99 & 49.96 & 49.61 & 50.18 \\
\hline $\mathrm{TiO}_{2}$ & 1.50 & 1.65 & 1.77 & 1.57 & 1.17 & 1.10 & 1.20 & 1.17 & 1.18 & 1.14 \\
\hline $\mathrm{Al}_{2} \mathrm{O}_{3}$ & 3.30 & 3.52 & 3.72 & 3.61 & 3.66 & 3.73 & 3.76 & 4.02 & 4.37 & 3.79 \\
\hline $\mathrm{Cr}_{2} \mathrm{O}_{3}$ & 0.00 & 0.02 & 0.06 & 0.07 & 0.35 & 0.62 & 0.35 & 0.69 & 0.92 & 0.49 \\
\hline $\mathrm{Fe}_{2} \mathrm{O}_{3}$ & 3.07 & 3.37 & 2.77 & 3.02 & 2.26 & 2.49 & 2.44 & 1.99 & 2.21 & 2.28 \\
\hline $\mathrm{FeO}$ & 5.46 & 5.23 & 5.48 & 4.60 & 4.31 & 4.14 & 4.33 & 4.24 & 3.80 & 4.35 \\
\hline $\mathrm{MnO}$ & 0.18 & 0.15 & 0.14 & 0.13 & 0.10 & 0.12 & 0.09 & 0.06 & 0.09 & 0.14 \\
\hline $\mathrm{MgO}$ & 14.79 & 14.89 & 14.65 & 15.23 & 15.53 & 15.55 & 15.44 & 15.56 & 15.45 & 15.50 \\
\hline $\mathrm{CaO}$ & 20.94 & 21.00 & 21.00 & 21.35 & 21.31 & 21.30 & 21.40 & 21.39 & 21.59 & 21.63 \\
\hline $\mathrm{Na}_{2} \mathrm{O}$ & 0.45 & 0.46 & 0.40 & 0.34 & 0.32 & 0.34 & 0.32 & 0.30 & 0.28 & 0.26 \\
\hline Total & 99.52 & 99.89 & 99.26 & 99.64 & 99.01 & 99.45 & 99.32 & 99.37 & 99.51 & 99.76 \\
\hline $\mathrm{Si}$ & 1.86 & 1.84 & 1.84 & 1.85 & 1.86 & 1.86 & 1.86 & 1.85 & 1.84 & 1.86 \\
\hline $\mathrm{Ti}$ & 0.04 & 0.05 & 0.05 & 0.04 & 0.03 & 0.03 & 0.03 & 0.03 & 0.03 & 0.03 \\
\hline $\mathrm{Al}$ & 0.15 & 0.15 & 0.16 & 0.16 & 0.16 & 0.16 & 0.16 & 0.18 & 0.19 & 0.17 \\
\hline $\mathrm{Cr}$ & 0.00 & 0.00 & 0.00 & 0.00 & 0.01 & 0.02 & 0.01 & 0.02 & 0.03 & 0.01 \\
\hline $\mathrm{Fe}^{3+}$ & 0.09 & 0.09 & 0.08 & 0.08 & 0.06 & 0.07 & 0.07 & 0.06 & 0.06 & 0.06 \\
\hline $\mathrm{Fe}^{2+}$ & 0.17 & 0.16 & 0.17 & 0.14 & 0.13 & 0.13 & 0.13 & 0.13 & 0.12 & 0.13 \\
\hline $\mathrm{Mn}$ & 0.01 & 0.00 & 0.00 & 0.00 & 0.00 & 0.00 & 0.00 & 0.00 & 0.00 & 0.00 \\
\hline $\mathrm{Mg}$ & 0.82 & 0.83 & 0.82 & 0.84 & 0.86 & 0.86 & 0.85 & 0.86 & 0.85 & 0.85 \\
\hline $\mathrm{Ca}$ & 0.84 & 0.84 & 0.84 & 0.85 & 0.85 & 0.85 & 0.85 & 0.85 & 0.86 & 0.86 \\
\hline $\mathrm{Na}$ & 0.03 & 0.03 & 0.03 & 0.02 & 0.02 & 0.02 & 0.02 & 0.02 & 0.02 & 0.02 \\
\hline Total & 4.00 & 4.00 & 4.00 & 4.00 & 4.00 & 4.00 & 4.00 & 4.00 & 4.00 & 4.00 \\
\hline $\mathrm{Fe}[\mathrm{t}]+\mathrm{Mn}$ & 13.64 & 13.61 & 13.28 & 12.03 & 10.49 & 10.56 & 10.75 & 9.93 & 9.63 & 10.59 \\
\hline $\mathrm{Mg \#}$ & 75.84 & 75.92 & 76.29 & 78.46 & 81.12 & 81.02 & 80.62 & 82.02 & 82.40 & 80.83 \\
\hline Wo & 36.89 & 36.46 & 36.72 & 36.99 & 37.21 & 36.65 & 37.09 & 36.72 & 36.35 & 37.25 \\
\hline En & 41.12 & 41.29 & 40.85 & 42.18 & 43.08 & 43.00 & 42.75 & 43.01 & 42.66 & 42.73 \\
\hline Fs & 8.78 & 8.11 & 8.80 & 7.36 & 6.87 & 6.59 & 6.85 & 6.63 & 6.01 & 6.93 \\
\hline Sample-unit & \multicolumn{10}{|c|}{ June Complex } \\
\hline Rock-type & \multicolumn{10}{|c|}{ Talc-bearing greenschist } \\
\hline Location & \multicolumn{6}{|c|}{ Clinopyroxene-rim } & \multicolumn{4}{|c|}{ Clinopyroxene-core } \\
\hline $\mathrm{SiO}_{2}$ & 50.05 & 50.07 & 50.24 & 49.95 & 49.69 & 50.53 & 52.08 & 52.10 & 52.13 & 51.83 \\
\hline $\mathrm{TiO}_{2}$ & 1.17 & 1.04 & 0.97 & 1.07 & 1.18 & 0.83 & 0.50 & 0.50 & 0.46 & 0.51 \\
\hline $\mathrm{Al}_{2} \mathrm{O}_{3}$ & 3.89 & 4.02 & 3.74 & 4.00 & 4.23 & 3.79 & 2.29 & 2.30 & 2.27 & 2.31 \\
\hline $\mathrm{Cr}_{2} \mathrm{O}_{3}$ & 0.49 & 0.73 & 0.65 & 0.73 & 0.51 & 0.94 & 1.00 & 0.98 & 1.10 & 0.83 \\
\hline $\mathrm{Fe}_{2} \mathrm{O}_{3}$ & 2.04 & 1.80 & 2.11 & 2.23 & 2.11 & 2.22 & 1.55 & 1.42 & 1.21 & 2.06 \\
\hline $\mathrm{FeO}$ & 4.35 & 4.15 & 3.75 & 3.70 & 4.18 & 3.33 & 2.75 & 2.82 & 3.02 & 2.24 \\
\hline $\mathrm{MnO}$ & 0.08 & 0.13 & 0.12 & 0.11 & 0.07 & 0.06 & 0.11 & 0.08 & 0.05 & 0.02 \\
\hline $\mathrm{MgO}$ & 15.54 & 15.62 & 15.85 & 15.74 & 15.49 & 16.01 & 17.41 & 17.35 & 17.28 & 17.43 \\
\hline $\mathrm{CaO}$ & 21.38 & 21.49 & 21.52 & 21.61 & 21.40 & 21.93 & 21.70 & 21.72 & 21.66 & 21.88 \\
\hline $\mathrm{Na}_{2} \mathrm{O}$ & 0.30 & 0.25 & 0.27 & 0.25 & 0.26 & 0.24 & 0.22 & 0.24 & 0.24 & 0.24 \\
\hline Total & 99.29 & 99.30 & 99.23 & 99.38 & 99.11 & 99.89 & 99.61 & 99.51 & 99.41 & 99.34 \\
\hline $\mathrm{Si}$ & 1.86 & 1.86 & 1.86 & 1.85 & 1.85 & 1.86 & 1.91 & 1.91 & 1.91 & 1.90 \\
\hline $\mathrm{Ti}$ & 0.03 & 0.03 & 0.03 & 0.03 & 0.03 & 0.02 & 0.01 & 0.01 & 0.01 & 0.01 \\
\hline $\mathrm{Al}$ & 0.17 & 0.18 & 0.16 & 0.17 & 0.19 & 0.16 & 0.10 & 0.10 & 0.10 & 0.10 \\
\hline
\end{tabular}


Table 2 continued

\begin{tabular}{|c|c|c|c|c|c|c|c|c|c|c|}
\hline \multirow{3}{*}{$\begin{array}{l}\text { Sample-unit } \\
\text { Rock-type } \\
\text { Location }\end{array}$} & \multicolumn{10}{|c|}{ June Complex } \\
\hline & \multicolumn{10}{|c|}{ Talc-bearing greenschist } \\
\hline & \multicolumn{6}{|c|}{ Clinopyroxene-rim } & \multicolumn{4}{|c|}{ Clinopyroxene-core } \\
\hline $\mathrm{Cr}$ & 0.01 & 0.02 & 0.02 & 0.02 & 0.02 & 0.03 & 0.03 & 0.03 & 0.03 & 0.02 \\
\hline $\mathrm{Fe}^{3+}$ & 0.06 & 0.05 & 0.06 & 0.06 & 0.06 & 0.06 & 0.04 & 0.04 & 0.03 & 0.06 \\
\hline $\mathrm{Fe}^{2+}$ & 0.14 & 0.13 & 0.12 & 0.11 & 0.13 & 0.10 & 0.08 & 0.09 & 0.09 & 0.07 \\
\hline Mn & 0.00 & 0.00 & 0.00 & 0.00 & 0.00 & 0.00 & 0.00 & 0.00 & 0.00 & 0.00 \\
\hline $\mathrm{Mg}$ & 0.86 & 0.86 & 0.88 & 0.87 & 0.86 & 0.88 & 0.95 & 0.95 & 0.95 & 0.95 \\
\hline $\mathrm{Ca}$ & 0.85 & 0.85 & 0.85 & 0.86 & 0.85 & 0.86 & 0.85 & 0.85 & 0.85 & 0.86 \\
\hline $\mathrm{Na}$ & 0.02 & 0.02 & 0.02 & 0.02 & 0.02 & 0.02 & 0.02 & 0.02 & 0.02 & 0.02 \\
\hline Total & 4.00 & 4.00 & 4.00 & 4.00 & 4.00 & 4.00 & 4.00 & 4.00 & 4.00 & 4.00 \\
\hline $\mathrm{Fe}[\mathrm{t}]+\mathrm{Mn}$ & 10.21 & 9.63 & 9.36 & 9.45 & 10.05 & 8.70 & 6.73 & 6.64 & 6.63 & 6.49 \\
\hline $\mathrm{Mg \#}$ & 81.56 & 82.52 & 83.05 & 82.83 & 81.79 & 84.10 & 87.96 & 88.11 & 88.11 & 88.33 \\
\hline Wo & 37.01 & 36.95 & 37.17 & 36.86 & 36.59 & 37.34 & 38.74 & 38.91 & 38.95 & 38.95 \\
\hline En & 42.99 & 43.18 & 43.80 & 43.46 & 42.91 & 43.92 & 47.58 & 47.45 & 47.31 & 47.71 \\
\hline Fs & 6.84 & 6.63 & 5.99 & 5.88 & 6.59 & 5.22 & 4.38 & 4.44 & 4.71 & 3.45 \\
\hline
\end{tabular}

$\mathrm{Mg} \#=100 \times \mathrm{Mg} / 100 \times \mathrm{Mg} /(\mathrm{Mg}+\mathrm{Fe}[\mathrm{t}]+\mathrm{Mn})$

Wo wollastonite, En enstatite, $F$ s ferrosillite

but all samples are low in $\mathrm{MnO}(\leq 0.2 \mathrm{wt} \%)$ and $\mathrm{P}_{2} \mathrm{O}_{5}$ $(\leq 0.4 \mathrm{wt} \%)$ contents. The investigated samples except one metagabbroic greenschist sample (P-125; LOI $\geq 6 \mathrm{wt} \%)$ have a loss on ignition (LOI) values of 1.19-5.82 wt \% $($ mean $=3.51 \mathrm{wt} \%)$, reflecting a minor contribution of secondary hydrated and carbonate phases (e.g., Polat and Hofmann 2003; Farahat 2011).

Mg-values $\left[\mathrm{Mg} \#=\mathrm{Mg} /\left(\mathrm{Mg}+\mathrm{Fe}^{2+}\right)\right]$ indicate two distinct groups of cumulates with $\mathrm{Mg \#}$ of 51.6-58.9 to basaltic liquids with Mg\# of 24.5-42.7 (Fig. 6a). Based on the total alkalies versus silica (TAS) diagram the magmatic protoliths of the studied rocks include peridotite-gabbro for cumulates, as well as monzo-gabbro and gabbro (Fig. 6b).

\subsection{Trace and rare earth elements}

Based on immobile elements, the mafic samples show a continuous range of compositions from alkaline to subalkaline basalt (Fig. 6c; Winchester and Floyd 1977). On the $\mathrm{Mg \#}$ vs. $\mathrm{SiO}_{2} / \mathrm{Al}_{2} \mathrm{O}_{3}$ diagram (Fig. 6d), the majority of the mafic rocks plots in the field of primitive basalt, which correlates with the alkaline and tholeiitic fractionation trend, whereas the cumulate mafic rocks $(\mathrm{Mg} \#>50)$ reveal a correlation with a compositional trend referenced by pyroxene accumulation.

The rare earth element (REE) contents of mafic samples (Fig. 7a) show that the analyzed samples, except for samples $\mathrm{P}-124, \mathrm{P}-125$ and $\mathrm{P}-105$, are similar to OIB patterns.
Most of the samples are REE enriched, likewise steep REE patterns with total REE concentrations $(64.15-155.95 \mathrm{ppm}),(\mathrm{La} / \mathrm{Yb})_{\mathrm{N}}$ ratios of 5.44-9.00, and no pronounced negative $\mathrm{Eu}$ anomaly $\left(\mathrm{Eu} / \mathrm{Eu}^{*}=+0.96\right.$ to $+1.61)$. The REE pattern of two fine-grained metagabbroic greenschists (P-124, P-125) and a talc-bearing greenschist $(\mathrm{P}-105)$ are different. They have lower $(\mathrm{La} / \mathrm{Yb})_{\mathrm{N}}$ ratio (2.48-3.70) and show E-type MORB-like trace element patterns. In the primitive mantle-normalized multi-element diagram (Fig. 7b) samples are characterized by enrichments in large-ion lithophile elements (LILE) relative to high field strength elements (HFSE). Nb shows either positive or no anomaly except for a fine-grained metagabbroic greenschist (P-125) that shows a negative anomaly (Fig. $7 \mathrm{~b}$ ). The positive $\mathrm{Nb}$ anomalies have been explained in terms of magma generation at plume source containing slab material recycled at greatest mantle depths (Kerrich and Xie 2002). Compared to cumulate samples, the other samples have slight positive $\mathrm{Ba}, \mathrm{Sr}$ and $\mathrm{Pb}$ anomalies. The slight positive $\mathrm{Sr}, \mathrm{Ba}$ and $\mathrm{Eu}$ anomalies can be caused by accumulation of plagioclase (Blundy and Wood 1991).

\section{$7 \mathrm{Sr}-\mathrm{Nd}$ isotopes}

Whole-rock $\mathrm{Rb}-\mathrm{Sr}$ and $\mathrm{Sm}-\mathrm{Nd}$ isotopic data for the Late Triassic an actinolite-bearing greenschist (P-147) is given in Table 5. The sample has a ${ }^{87} \mathrm{Sr} /{ }^{86} \mathrm{Sr}$ initial ratio of 0.706 
Table 3 Representative analyses of amphibole, plagioclase and K-feldspar from the June Complex

\begin{tabular}{|c|c|c|c|c|c|c|c|c|c|c|c|c|c|c|}
\hline \multirow{3}{*}{$\begin{array}{l}\text { Unit } \\
\text { Sample no. and type } \\
\text { Mineral }\end{array}$} & \multicolumn{14}{|c|}{ June Complex } \\
\hline & \multicolumn{10}{|c|}{ P-147-actinolite-bearing greenschist } & \multicolumn{4}{|c|}{ P-149-talc-bearing greenschist } \\
\hline & Amph & & & & & & & & & & Amph & & & \\
\hline $\mathrm{SiO}_{2}$ & 43.91 & 43.33 & 56.84 & 55.56 & 56.92 & 52.56 & 51.52 & 47.70 & 44.66 & 57.25 & 56.51 & 57.43 & 53.36 & 52.92 \\
\hline $\mathrm{Al}_{2} \mathrm{O}_{3}$ & 8.70 & 9.31 & 0.20 & 1.34 & 0.59 & 3.80 & 4.36 & 7.03 & 8.85 & 0.43 & 1.26 & 2.80 & 3.79 & 3.06 \\
\hline $\mathrm{MgO}$ & 14.37 & 14.23 & 19.28 & 19.13 & 19.29 & 15.27 & 15.22 & 14.45 & 14.40 & 19.56 & 14.34 & 15.88 & 16.43 & 17.51 \\
\hline $\mathrm{Na}_{2} \mathrm{O}$ & 2.64 & 2.70 & 0.05 & 0.62 & 0.08 & 0.68 & 0.79 & 1.67 & 2.70 & 0.27 & 0.42 & 1.14 & 0.90 & 0.19 \\
\hline $\mathrm{CaO}$ & 10.87 & 11.07 & 13.11 & 12.31 & 13.00 & 12.60 & 12.65 & 11.58 & 10.94 & 12.74 & 12.36 & 11.62 & 12.06 & 10.97 \\
\hline $\mathrm{TiO}_{2}$ & 2.78 & 3.39 & 0.02 & 0.02 & 0.02 & 0.03 & 0.07 & 2.56 & 2.89 & 0.05 & 0.08 & 0.01 & 0.07 & 0.07 \\
\hline $\mathrm{FeO}$ & 11.35 & 12.33 & 7.54 & 7.76 & 7.81 & 12.14 & 11.27 & 11.30 & 11.57 & 6.24 & 9.47 & 9.10 & 11.08 & 12.01 \\
\hline $\mathrm{MnO}$ & 0.20 & 0.26 & 0.16 & 0.16 & 0.15 & 0.23 & 0.22 & 0.18 & 0.22 & 0.17 & 0.13 & 0.16 & 0.14 & 0.15 \\
\hline $\mathrm{Cr}_{2} \mathrm{O}_{3}$ & 0.03 & 0.00 & 0.03 & 0.00 & 0.00 & 0.07 & 0.71 & 0.00 & 0.00 & 0.01 & 0.00 & 0.00 & 0.00 & 0.05 \\
\hline $\mathrm{K}_{2} \mathrm{O}$ & 0.71 & 0.72 & 0.00 & 0.05 & 0.00 & 0.07 & 0.08 & 0.55 & 0.74 & 0.00 & 0.00 & 0.00 & 0.03 & 0.00 \\
\hline Total & 95.55 & 97.33 & 97.24 & 96.96 & 97.86 & 97.45 & 96.88 & 97.03 & 96.97 & 96.73 & 94.57 & 98.14 & 97.86 & 96.92 \\
\hline Per $\mathrm{O}$ & 23 & 23 & 23 & 23 & 23 & 23 & 23 & 23 & 23 & 23 & 23 & 23 & 23 & 23 \\
\hline $\mathrm{Si}$ & 6.59 & 6.43 & 8.00 & 7.86 & 7.97 & 7.59 & 7.48 & 6.98 & 6.61 & 8.04 & 8.20 & 8.02 & 7.61 & 7.62 \\
\hline $\mathrm{Al}$ & 1.54 & 1.63 & 0.03 & 0.22 & 0.10 & 0.65 & 0.75 & 1.21 & 1.54 & 0.07 & 0.22 & 0.46 & 0.64 & 0.52 \\
\hline $\mathrm{Mg}$ & 3.22 & 3.15 & 4.05 & 4.04 & 4.02 & 3.29 & 3.30 & 3.15 & 3.18 & 4.09 & 3.10 & 3.30 & 3.49 & 3.76 \\
\hline $\mathrm{Na}$ & 0.77 & 0.78 & 0.01 & 0.17 & 0.02 & 0.19 & 0.22 & 0.47 & 0.78 & 0.07 & 0.12 & 0.31 & 0.25 & 0.05 \\
\hline $\mathrm{Ca}$ & 1.75 & 1.76 & 1.98 & 1.87 & 1.95 & 1.95 & 1.97 & 1.82 & 1.73 & 1.92 & 1.92 & 1.74 & 1.84 & 1.69 \\
\hline $\mathrm{Ti}$ & 0.31 & 0.38 & 0.00 & 0.00 & 0.00 & 0.00 & 0.01 & 0.28 & 0.32 & 0.01 & 0.01 & 0.00 & 0.01 & 0.01 \\
\hline $\mathrm{Fe}$ & 1.43 & 1.53 & 0.89 & 0.92 & 0.91 & 1.47 & 1.37 & 1.38 & 1.43 & 0.73 & 1.15 & 1.06 & 1.32 & 1.45 \\
\hline $\mathrm{Mn}$ & 0.02 & 0.03 & 0.02 & 0.02 & 0.02 & 0.03 & 0.03 & 0.02 & 0.03 & 0.02 & 0.02 & 0.02 & 0.02 & 0.02 \\
\hline $\mathrm{Cr}$ & 0.00 & 0.00 & 0.00 & 0.00 & 0.00 & 0.01 & 0.08 & 0.00 & 0.00 & 0.00 & 0.00 & 0.00 & 0.00 & 0.01 \\
\hline $\mathrm{K}$ & 0.14 & 0.14 & 0.00 & 0.01 & 0.00 & 0.01 & 0.01 & 0.10 & 0.14 & 0.00 & 0.00 & 0.00 & 0.00 & 0.00 \\
\hline Total & 38.77 & 38.83 & 37.98 & 38.11 & 37.99 & 38.18 & 38.22 & 38.42 & 38.76 & 37.96 & 37.74 & 37.91 & 38.19 & 38.13 \\
\hline $\mathrm{Al}^{\mathrm{IV}}$ & 1.46 & 1.59 & 0.00 & 0.16 & 0.03 & 0.45 & 0.55 & 0.15 & 1.05 & 1.40 & 0.00 & 0.00 & 0.43 & 0.43 \\
\hline $\mathrm{Al}^{\mathrm{VI}}$ & 0.07 & 0.03 & 0.03 & 0.06 & 0.07 & 0.19 & 0.19 & 0.49 & 0.15 & 0.08 & 0.21 & 0.46 & 0.30 & 0.08 \\
\hline $\mathrm{Mg \#}$ & 0.69 & 0.67 & 0.82 & 0.81 & 0.81 & 0.69 & 0.71 & 0.70 & 0.69 & 0.85 & 0.73 & 0.76 & 0.73 & 0.72 \\
\hline Unit & \multicolumn{14}{|c|}{ June Complex } \\
\hline Sample no. and type & \multicolumn{6}{|c|}{ P-149-talc-bearing greenschist } & \multicolumn{8}{|c|}{ P-119-metagabbroic greenschists } \\
\hline Mineral & Amph & & & $\mathrm{Pl}$ & & Kfs & $\mathrm{Pl}$ & & & & Amph & & & \\
\hline $\mathrm{SiO}_{2}$ & 55.05 & 53.55 & 55.39 & 64.21 & 67.39 & 64.96 & 60.23 & 57.69 & 58.45 & 59.87 & 44.87 & 45.29 & 47.81 & 46.92 \\
\hline $\mathrm{Al}_{2} \mathrm{O}_{3}$ & 2.05 & 2.24 & 1.25 & 20.05 & 19.21 & 18.10 & 24.37 & 25.93 & 25.46 & 24.63 & 11.67 & 10.95 & 9.12 & 9.38 \\
\hline $\mathrm{MgO}$ & 13.95 & 14.64 & 14.14 & 1.08 & 0.56 & 0.27 & 0.00 & 0.00 & 0.00 & 0.03 & 12.67 & 12.84 & 13.92 & 13.86 \\
\hline $\mathrm{Na}_{2} \mathrm{O}$ & 0.45 & 0.22 & 0.25 & 9.72 & 10.62 & 0.26 & 8.10 & 7.05 & 7.42 & 7.97 & 1.73 & 1.63 & 1.32 & 1.38 \\
\hline $\mathrm{CaO}$ & 12.51 & 12.30 & 12.42 & 1.13 & 0.25 & 0.14 & 6.09 & 7.93 & 7.35 & 6.43 & 11.50 & 11.70 & 11.60 & 11.69 \\
\hline $\mathrm{TiO}_{2}$ & 0.01 & 1.07 & 0.47 & 0.00 & 0.00 & 0.00 & 0.00 & 0.02 & 0.00 & 0.01 & 0.54 & 0.54 & 0.39 & 0.36 \\
\hline $\mathrm{FeO}$ & 10.24 & 10.15 & 10.37 & 1.64 & 0.75 & 0.75 & 0.00 & 0.09 & 0.04 & 0.00 & 14.00 & 13.92 & 12.99 & 12.51 \\
\hline $\mathrm{MnO}$ & 0.16 & 0.12 & 0.15 & 0.00 & 0.02 & 0.00 & 0.00 & 0.00 & 0.00 & 0.00 & 0.33 & 0.28 & 0.31 & 0.31 \\
\hline $\mathrm{Cr}_{2} \mathrm{O}_{3}$ & 0.04 & 0.04 & 0.00 & 0.03 & 0.00 & 0.00 & 0.00 & 0.00 & 0.00 & 0.00 & 0.06 & 0.07 & 0.01 & 0.00 \\
\hline $\mathrm{K}_{2} \mathrm{O}$ & 0.00 & 0.00 & 0.00 & 0.05 & 0.05 & 15.64 & 0.03 & 0.04 & 0.03 & 0.04 & 0.17 & 0.16 & 0.10 & 0.09 \\
\hline Total & 94.47 & 94.31 & 94.44 & 97.92 & 98.8 & 100.1 & 98.81 & 98.74 & 98.75 & 98.98 & 99.58 & 99.44 & 99.65 & 98.97 \\
\hline
\end{tabular}


Table 3 continued

\begin{tabular}{|c|c|c|c|c|c|c|c|c|c|c|c|c|c|c|}
\hline \multirow{3}{*}{$\begin{array}{l}\text { Unit } \\
\text { Sample no. and type } \\
\text { Mineral }\end{array}$} & \multicolumn{14}{|c|}{ June Complex } \\
\hline & \multicolumn{6}{|c|}{ P-149-talc-bearing greenschist } & \multicolumn{8}{|c|}{ P-119-metagabbroic greenschists } \\
\hline & Amph & & & $\mathrm{Pl}$ & & Kfs & $\mathrm{Pl}$ & & & & Amph & & & \\
\hline Per O & 23 & 23 & 23 & 32 & 32 & 32 & 8 & 8 & 8 & 8 & 24 & 24 & 24 & 24 \\
\hline $\mathrm{Si}$ & 8.06 & 7.87 & 8.11 & 11.6 & 11.93 & 11.99 & 2.71 & 2.61 & 2.64 & 2.69 & 6.54 & 6.61 & 6.91 & 6.83 \\
\hline $\mathrm{Al}$ & 0.35 & 0.39 & 0.22 & 4.3 & 4.01 & 3.94 & 1.29 & 1.38 & 1.36 & 1.30 & 2.00 & 1.88 & 1.55 & 1.61 \\
\hline $\mathrm{Mg}$ & 3.04 & 3.21 & 3.09 & 0.3 & 0.15 & 0.08 & 0.00 & 0.00 & 0.00 & 0.00 & 2.75 & 2.79 & 3.00 & 3.01 \\
\hline $\mathrm{Na}$ & 0.13 & 0.06 & 0.07 & 3.4 & 3.65 & 0.09 & 0.71 & 0.62 & 0.65 & 0.69 & 0.49 & 0.46 & 0.37 & 0.39 \\
\hline $\mathrm{Ca}$ & 1.96 & 1.94 & 1.95 & 0.2 & 0.05 & 0.03 & 0.29 & 0.38 & 0.36 & 0.31 & 1.80 & 1.83 & 1.80 & 1.82 \\
\hline $\mathrm{Ti}$ & 0.00 & 0.12 & 0.05 & 0.0 & 0.00 & 0.00 & 0.00 & 0.00 & 0.00 & 0.00 & 0.06 & 0.06 & 0.04 & 0.04 \\
\hline $\mathrm{Fe}$ & 1.25 & 1.25 & 1.27 & 0.2 & 0.11 & 0.12 & 0.00 & 0.00 & 0.00 & 0.00 & 1.64 & 1.64 & 1.52 & 1.50 \\
\hline $\mathrm{Mn}$ & 0.02 & 0.01 & 0.02 & 0.0 & 0.00 & 0.00 & 0.00 & 0.00 & 0.00 & 0.00 & 0.04 & 0.03 & 0.04 & 0.04 \\
\hline $\mathrm{Cr}$ & 0.00 & 0.00 & 0.00 & 0.0 & 0.00 & 0.00 & & & & & 0.01 & 0.01 & 0.00 & 0.00 \\
\hline K & 0.00 & 0.00 & 0.00 & 0.0 & 0.01 & 3.68 & 0.00 & 0.00 & 0.00 & 0.00 & 0.03 & 0.03 & 0.02 & 0.02 \\
\hline Total & 37.83 & 37.85 & 37.77 & 52.00 & 51.90 & 51.93 & 5.00 & 5.00 & 5.01 & 5.00 & 17.37 & 17.36 & 17.26 & 17.28 \\
\hline $\mathrm{Al}^{\mathrm{IV}}$ & 0.32 & 0.09 & 0.19 & & & & & & & & 1.45 & 1.38 & 1.08 & 1.16 \\
\hline $\mathrm{Al}^{\mathrm{VI}}$ & 0.00 & 0.30 & 0.00 & & & & & & & & 0.54 & 0.49 & 0.46 & 0.44 \\
\hline $\mathrm{Mg \#}$ & 0.71 & 0.72 & 0.71 & & & & & & & & 0.72 & 0.72 & 0.73 & 0.74 \\
\hline $\mathrm{X}_{\mathrm{Ab}}$ & & & & 0.936 & 0.984 & 0.024 & 0.71 & 0.62 & 0.64 & 0.69 & & & & \\
\hline$X_{\text {An }}$ & & & & 0.060 & 0.013 & 0.007 & 0.29 & 0.38 & 0.35 & 0.31 & & & & \\
\hline $\mathrm{X}_{\text {Or }}$ & & & & 0.003 & 0.003 & 0.968 & 0.00 & 0.00 & 0.00 & 0.00 & & & & \\
\hline
\end{tabular}

and $\varepsilon \mathrm{Nd}_{220}$ value of +1.002 . Considering the low ${ }^{87} \mathrm{Sr} /{ }^{86} \mathrm{Sr}$ and $\varepsilon \mathrm{Nd}_{220}$ value above that of bulk earth, suggest an enriched mantle source with a relatively low degree of crustal contamination for the cumulate mafic magmas (Fig. 8).

\section{Discussion}

Fossils indicate a Triassic age of the June Complex and these also infer a Triassic age for the basaltic rocks, particularly because of the interlayering of greenschists and metasediments (Fig. 2a, b). The boudinaged metagabbroic and talc-bearing greenschists could represent cumulates of sills.

\subsection{Magma source}

Geochemical characteristics in both cumulate and basaltic mafic rocks can be used to monitor mantle source compositions, the conditions of partial melting, and possibly shallow level processes during magma emplacement (e.g., fractional crystallization and/or crustal contamination). Thus, the role of fractional crystallization and crustal contamination should be initially assessed before addressing the nature of magma sources of these mafic rocks.
High concentrations of $\mathrm{Cr}$ (1073-1341 ppm) and $\mathrm{Ni}$ (584-709 ppm) in cumulate samples are in agreement with a mantle origin for the magma, which was enriched in pyroxene and olivine. This magma underwent fractional crystallization and accumulation of mafic minerals as shown by the Fig. $6 \mathrm{~d}$. The basaltic samples have lower $\mathrm{MgO}$ and compatible trace elements (e.g., Ni, Cr). This feature suggests that the studied samples experienced significant fractional crystallization and/or the source area was affected by different degrees of partial melting. All mafic samples yield a negative correlation of $\mathrm{MgO}$ and compatible elements (e.g., $\mathrm{Ni}, \mathrm{Cr}$ ) with increasing $\mathrm{Zr}$ contents and positive correlation of LREE, HREE and Nb (Fig. 9). This feature is consistent with the fractionation of olivine, pyroxene and plagioclase (e.g., Ordóñez-Calderón et al. 2011). In addition, $\mathrm{TiO}_{2}$ and $\mathrm{V}$ abundances increase with increasing $\mathrm{Zr}$ contents suggest that $\mathrm{Fe}-\mathrm{Ti}$ oxides (ilmenite and titanomagnetite) were not significant mineral phases during fractionation (e.g., Ordóñez-Calderón et al. 2011; Deng et al. 2013). Nevertheless, it is uncertain whether such covariations between the major and trace elements indicate fractional crystallization of basaltic magmas or reflect the nature of magma sources. However, the increasing $\mathrm{Zr} / \mathrm{Nb}(<10)$ ratios are accompanied by decreasing $\mathrm{Ce} / \mathrm{Y}$ ratios (not shown) pointing to a variable degree of partial melting rather than a fractional 
Table 4 Major (in wt $\%$ ) and trace element (in ppm) data for the mafic rocks from the June Complex

\begin{tabular}{|c|c|c|c|c|c|c|}
\hline \multirow[t]{2}{*}{ Sample no. } & \multicolumn{2}{|c|}{ Actinolite-bearing greenschist } & \multicolumn{4}{|c|}{ Metagabbroic greenschist (epidote-amphibolite) } \\
\hline & P147 & P154 & P119 & $\mathrm{P} 125$ & $\mathrm{P} 134$ & $\mathrm{~J} 212$ \\
\hline $\mathrm{SiO}_{2} \mathrm{wt} \%$ & 45.50 & 43.54 & 46.57 & 45.40 & 45.72 & 44.46 \\
\hline $\mathrm{Al}_{2} \mathrm{O}_{3}$ & 6.76 & 7.08 & 16.14 & 15.00 & 14.62 & 14.83 \\
\hline $\mathrm{Fe}_{2} \mathrm{O}_{3}$ & 12.09 & 12.38 & 11.62 & 12.59 & 12.30 & 10.50 \\
\hline $\mathrm{MgO}$ & 19.42 & 20.61 & 7.08 & 10.42 & 8.57 & 9.06 \\
\hline $\mathrm{CaO}$ & 9.67 & 8.79 & 10.55 & 6.85 & 9.20 & 10.30 \\
\hline $\mathrm{Na}_{2} \mathrm{O}$ & 0.62 & 0.20 & 3.16 & 1.48 & 2.94 & 2.96 \\
\hline $\mathrm{K}_{2} \mathrm{O}$ & 9.67 & 8.79 & 10.55 & 6.85 & 9.20 & 10.30 \\
\hline $\mathrm{TiO}_{2}$ & 1.25 & 1.31 & 2.81 & 1.14 & 2.28 & 2.67 \\
\hline $\mathrm{P}_{2} \mathrm{O}_{5}$ & 0.14 & 0.16 & 0.30 & 0.11 & 0.40 & 0.40 \\
\hline $\mathrm{MnO}$ & 0.16 & 0.15 & 0.19 & 0.10 & 0.23 & 0.15 \\
\hline LOI & 4.56 & 5.82 & 1.19 & 6.37 & 2.48 & 3.58 \\
\hline Total & 100.2 & 100.1 & 100.1 & 99.6 & 99.5 & 99.6 \\
\hline $\mathrm{Mg \#}$ & 58.1 & 58.9 & 34.4 & 41.6 & 37.5 & 42.7 \\
\hline $\mathrm{Li}(\mathrm{ppm})$ & 17.1 & 5.1 & 10.6 & 26.1 & 16.0 & 35.0 \\
\hline $\mathrm{Be}$ & 0.58 & 0.59 & 1.45 & 0.76 & 1.10 & 1.51 \\
\hline $\mathrm{Sc}$ & 32.8 & 29.8 & 36.3 & 29.9 & 28.4 & 28.1 \\
\hline V & 172 & 172 & 430 & 252 & 295 & 461 \\
\hline $\mathrm{Cr}$ & 1341 & 1217 & 317 & 438 & 265 & 411 \\
\hline Co & 76.8 & 76.7 & 44.8 & 54.1 & 48.5 & 43.4 \\
\hline $\mathrm{Ni}$ & 681 & 709 & 113 & 254 & 107 & 158 \\
\hline $\mathrm{Cu}$ & 32.4 & 103.3 & 44.9 & 80.4 & 88.3 & 42.6 \\
\hline $\mathrm{Zn}$ & 91 & 83 & 81 & 99 & 128 & 106 \\
\hline $\mathrm{Ga}$ & 10.7 & 10.3 & 23.6 & 19.2 & 19.2 & 19.4 \\
\hline $\mathrm{Ge}$ & 1.46 & 1.45 & 1.70 & 1.43 & 1.30 & 1.55 \\
\hline $\mathrm{Rb}$ & 0.88 & 0.80 & 13.07 & 3.61 & 17.58 & 19.94 \\
\hline $\mathrm{Sr}$ & 103 & 113 & 389 & 493 & 363 & 397 \\
\hline $\mathrm{Y}$ & 12.5 & 13.0 & 39.1 & 17.8 & 26.3 & 28.2 \\
\hline $\mathrm{Zr}$ & 80.4 & 83.1 & 187.2 & 55.4 & 173.6 & 210.4 \\
\hline $\mathrm{Nb}$ & 14.30 & 15.42 & 23.49 & 6.44 & 35.42 & 44.36 \\
\hline Cs & 0.13 & 0.10 & 0.24 & 0.34 & 0.35 & 0.50 \\
\hline $\mathrm{Ba}$ & 4.2 & 7.2 & 68.9 & 80.1 & 238.8 & 183.5 \\
\hline $\mathrm{La}$ & 11.54 & 12.52 & 25.48 & 5.69 & 22.98 & 27.14 \\
\hline $\mathrm{Ce}$ & 24.31 & 25.70 & 55.25 & 12.58 & 48.49 & 60.82 \\
\hline $\operatorname{Pr}$ & 3.20 & 3.42 & 7.35 & 1.77 & 6.16 & 7.97 \\
\hline $\mathrm{Nd}$ & 12.88 & 13.42 & 31.02 & 7.91 & 24.75 & 32.26 \\
\hline $\mathrm{Sm}$ & 2.81 & 2.90 & 7.04 & 2.22 & 5.31 & 6.75 \\
\hline $\mathrm{Eu}$ & 1.05 & 0.89 & 3.72 & 1.27 & 1.78 & 2.07 \\
\hline $\mathrm{Gd}$ & 2.72 & 2.78 & 7.12 & 2.70 & 5.20 & 6.23 \\
\hline $\mathrm{Tb}$ & 0.42 & 0.43 & 1.12 & 0.48 & 0.80 & 0.92 \\
\hline Dy & 2.38 & 2.39 & 6.66 & 3.07 & 4.72 & 5.22 \\
\hline Но & 0.44 & 0.45 & 1.31 & 0.62 & 0.89 & 0.98 \\
\hline $\mathrm{Er}$ & 1.16 & 1.20 & 3.60 & 1.71 & 2.44 & 2.63 \\
\hline $\mathrm{Tm}$ & 0.16 & 0.16 & 0.51 & 0.24 & 0.35 & 0.37 \\
\hline $\mathrm{Yb}$ & 0.96 & 0.99 & 3.16 & 1.49 & 2.13 & 2.27 \\
\hline $\mathrm{Lu}$ & 0.13 & 0.13 & 0.45 & 0.21 & 0.30 & 0.32 \\
\hline $\mathrm{Hf}$ & 1.91 & 1.99 & 3.90 & 1.40 & 3.62 & 4.51 \\
\hline $\mathrm{Ta}$ & 0.82 & 0.88 & 1.35 & 0.37 & 1.88 & 2.43 \\
\hline $\mathrm{Pb}$ & 0.41 & 0.51 & 5.74 & 1.32 & 2.59 & 7.02 \\
\hline
\end{tabular}


Table 4 continued

\begin{tabular}{|c|c|c|c|c|c|c|}
\hline \multirow[t]{2}{*}{ Sample no. } & \multicolumn{2}{|c|}{ Actinolite-bearing greenschist } & \multicolumn{4}{|c|}{ Metagabbroic greenschist (epidote-amphibolite) } \\
\hline & P147 & P154 & P119 & $\mathrm{P} 125$ & P134 & $\mathrm{J} 212$ \\
\hline Th & 1.22 & 1.30 & 1.64 & 0.68 & 1.96 & 2.81 \\
\hline $\mathrm{U}$ & 0.27 & 0.32 & 1.44 & 0.18 & 0.60 & 1.39 \\
\hline
\end{tabular}
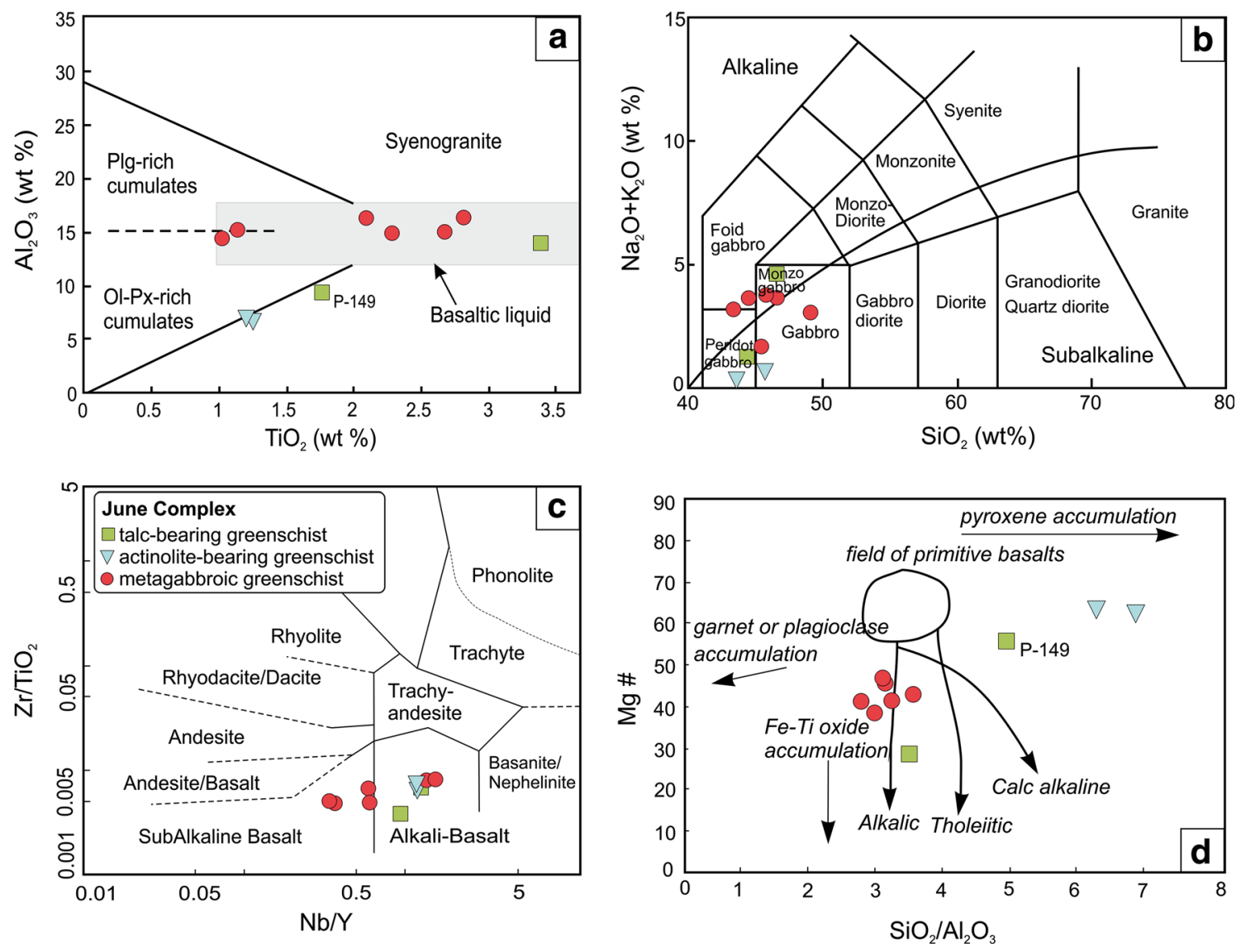

Fig. 6 Petrochemical classifications and characteristics of the studied mafic rocks. a $\mathrm{TiO}_{2}$ vs. $\mathrm{Al}_{2} \mathrm{O}_{3}$ diagram (Pearce 1983) showing the discrimination between basaltic liquids and cumulate. b Total alkali vs. Silica diagram of Le Maitre et al. (1989) with subalkaline-alkaline fields after Miyashiro (1978) displays the compositional range of the

crystallization process (e.g., Baker et al. 1997; Xia et al. 2004; Viccaro and Cristofolini 2008; Saccani et al. 2014).

Similar inferences are derived from the $(\mathrm{La} / \mathrm{Sm})_{\mathrm{PM}}$ vs. $(\mathrm{Tb} / \mathrm{Yb})_{\mathrm{PM}}$ diagram (Fig. 10a). The cumulate mafic samples plot above the $\mathrm{Tb} / \mathrm{Yb}$ discrimination line indicative of melting of garnet peridotite mantle at depth $>90 \mathrm{~km}$, whereas isotropic mafic samples derive from shallower spinel-peridotite mantle melting at a depth $<90 \mathrm{~km}$ (Herzberg 1995; Wang et al. 2002; Khudoley et al. 2013). Except for samples P-124, P-125 (fine-grained metagabbroic greenschists) and P-105 (talc-bearing greenschist) that show moderately low $(\mathrm{La} / \mathrm{Sm})_{\mathrm{PM}}$ ratios, all the other samples have higher $(\mathrm{La} / \mathrm{Sm})_{\mathrm{PM}}$ ratios. Khudoley et al.

magmatic protoliths. $\mathrm{c} \mathrm{Zr} / \mathrm{TiO}_{2} \times 0.0001-\mathrm{Nb} / \mathrm{Yb}$ classification diagram. d $\mathrm{Mg} \#$ vs. $\mathrm{SiO}_{2} / \mathrm{Al}_{2} \mathrm{O}_{3}$ diagram showing both the field of tholeiitic and alkali differentiation trends (Irvine and Baragar 1972) and mineral fractionation (after Kempton and Harmon 1992)

(2013) suggest that this difference can be caused by three processes, namely crustal contamination or increased partial melting, both of which can increase $\mathrm{La} / \mathrm{Sm}$ ratios in a melt, or melting of a depleted source, which would lower $\mathrm{La} / \mathrm{Sm}$ ratios. In the $(\mathrm{Th} / \mathrm{Yb})_{\mathrm{PM}} \mathrm{vs}$. $(\mathrm{Nb} / \mathrm{Yb})_{\mathrm{PM}}$ diagram (Fig. 10b) all mafic samples fall into the field of mantle melts. Moreover, all samples lie close to E-MORB and OIB, indicating insignificant crustal contamination (Fig. 10c). These diagrams clearly rule out significant crustal contamination and suggest a control of composition of mafic rocks by differences in the source region during mantle melting. Therefore, the differences in $\mathrm{La} / \mathrm{Sm}$ ratios is likely due to various degrees of partial melting, 

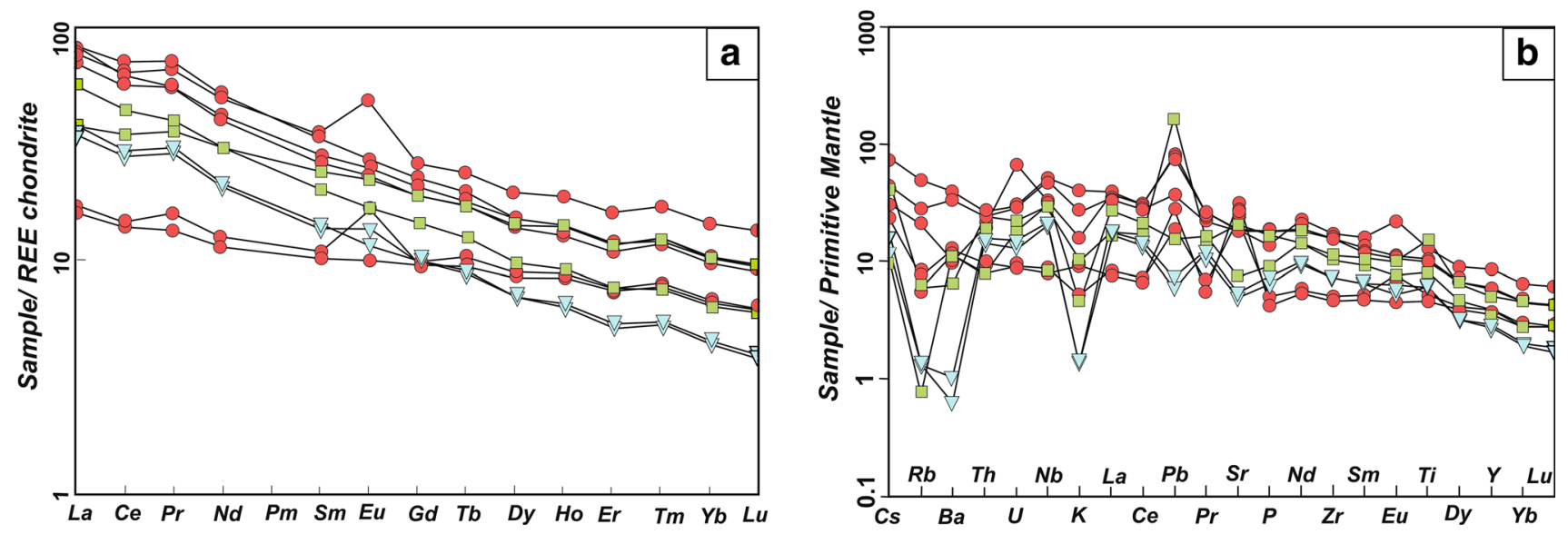

Fig. 7 a Chondrite-normalized (Boynton 1984) REE patterns and b primitive-mantle normalized incompatible trace element patterns of the analyzed mafic rocks (Sun and McDonough 1989). Symbols are as in Fig. 6

indicating that most mafic magmas being generated by partial melting at greater depth. This led to the formation of alkaline basalts, rather than tholeiitic basalts, with shallower partial melting. In addition, elevated $(\mathrm{Nb} / \mathrm{Yb})_{\mathrm{PM}}$ ratios (Fig. 10b) in the alkaline mafic samples potentially indicate mixing between magmas derived from both enriched and depleted mantle sources (Khudoley et al. 2013; Prokopiev et al. 2016).

\subsection{Crystallization conditions}

The composition of pyroxene, plagioclase and hornblende can be used to evaluate the chemical variation of magma and to determine magmatic P-T conditions. In the metagabbroic greenschists, temperature and pressure were calculated with the PET software (Dachs 2004). These calibrations yield crystallization temperatures and pressures for magmatic amphiboles at $690 \pm 30{ }^{\circ} \mathrm{C}$ and $5.6 \pm 0.8$ kbar.

In addition, the relation between $\mathrm{Al}$ in the tetrahedral sites and the Ti content in clinopyroxene can be used to discriminate geotectonic settings (Loucks 1990; Himmelberg and Loney 1995). Most clinopyroxenes of actinoliteand talc-bearing greenschists follow the rift-related cumulate trend (Fig. 11).

Here we use clinopyroxene of talc-bearing and actinolitebearing greenschists for estimation of $\mathrm{P}-\mathrm{T}$ conditions of magmatic mineral growth. The magmatic clinopyroxene of the basaltic talc-bearing greenschist (P-105) shows a sudden increase in the $\mathrm{Al}$ content in the rim of clinopyroxene (Fig. 5a, b). The $\mathrm{Al}$ concentration in clinopyroxene is controlled either by reaction of $\mathrm{NaAlSi}_{3} \mathrm{O}_{8}=\mathrm{NaAlSi}_{2} \mathrm{O}_{6}$ $+\mathrm{SiO}_{2}$ (a) at high pressure ( $>3.5 \mathrm{GPa}$, Kushiro 1969) or $\mathrm{CaAl}_{2} \mathrm{Si}_{2} \mathrm{O}_{8}=\mathrm{CaAl}_{2} \mathrm{SiO}_{6}+\mathrm{SiO}_{2}$ (b) at low pressure (0.5-1.2 GPa, Green 1967). Zhu and Ogasawara (2004) have suggested that the reaction (a) could happen only at depths
$>120 \mathrm{~km}$ (in a deep magma chamber), which would be recorded in the $\mathrm{Al}$ content of the diopside cores. The low $\mathrm{Al}$ concentration in diopside cores may indicate that they grew at a depth less than $120 \mathrm{~km}$ where reaction (a) occurred. A general decrease in $\mathrm{Fe}^{3+}$ in the core of clinopyroxene with increasing temperature is observed, too (Woodland 2009). We relate these changes to the evolution within the precursor magma within a magma chamber.

The empirical thermometer for clinopyroxene and amphibole of Perchuck et al. (1985) applied to clinopyroxene overgrown by magmatic hornblende of talc-bearing greenschists gives temperatures ranging between $800-1080{ }^{\circ} \mathrm{C}$ in the core and $650-780{ }^{\circ} \mathrm{C}$ in the rim.

In talc-bearing greenschists, the single-grain $\mathrm{Cpx}$ thermobarometry of Nimis and Taylor (2000) gives a similar temperature $\left(880-1000{ }^{\circ} \mathrm{C}\right)$. The diopsidic cores (P-149) yield a higher pressure (ca. $6 \mathrm{kbar}$ ) than the augitic rim (ca. $1.2 \mathrm{kbar}$ ), although some rim compositions yield moderately negative $\mathrm{P}$ values interpreted as recording Cpx crystallized at shallow levels (Nimis, personal communication).

It has to be noted that the source of cumulate talcbearing greenschist occur in the stability field of the garnet peridotite mantle (Fig. 10a).

\subsection{Tectonic setting implications}

The studied Upper Triassic mafic rocks display a range of compositions from subalkaline to alkaline basaltic magmas and show a transitional to alkaline signature (Fig. 6d). On the $\mathrm{Zr}$ vs. Y diagram (Fig. 12a), regression lines for nearly all samples have $\mathrm{Zr} / \mathrm{Y}$ ratios of 3-7, indicating an affinity with transitional and tholeiitic magmas. Almost all samples plot either in or close to the MORB + OIB subfield (Fig. 12b). The ratios of $\mathrm{Th} / \mathrm{Yb}$ and $\mathrm{Ta} / \mathrm{Yb}$ are largely independent of variations caused by the degree of partial melting and crystal fractionation, and thus essentially 

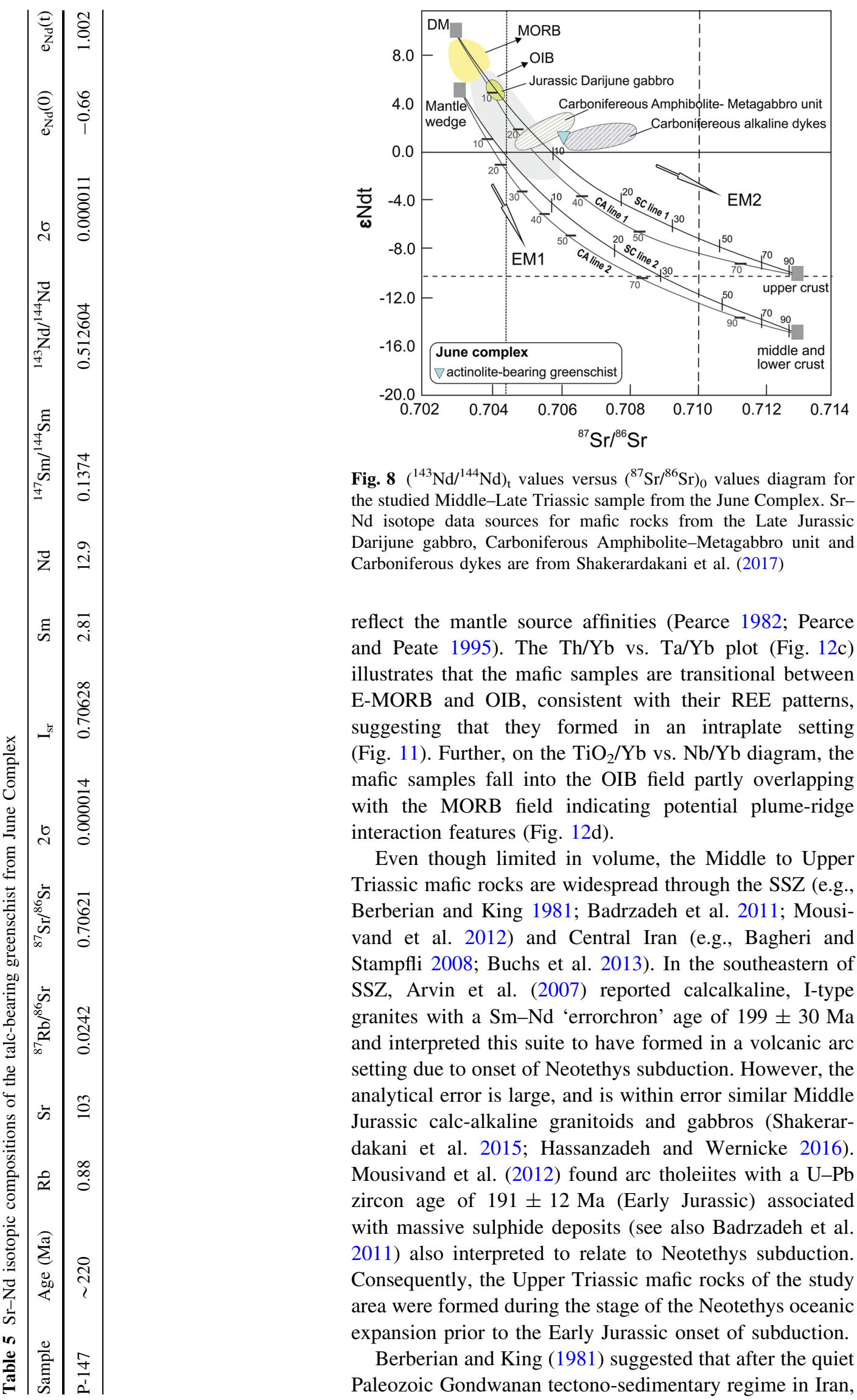

Fig. $8\left({ }^{143} \mathrm{Nd} /{ }^{144} \mathrm{Nd}\right)_{\mathrm{t}}$ values versus $\left({ }^{87} \mathrm{Sr} /{ }^{86} \mathrm{Sr}\right)_{0}$ values diagram for the studied Middle-Late Triassic sample from the June Complex. Sr$\mathrm{Nd}$ isotope data sources for mafic rocks from the Late Jurassic Darijune gabbro, Carboniferous Amphibolite-Metagabbro unit and Carboniferous dykes are from Shakerardakani et al. (2017)

reflect the mantle source affinities (Pearce 1982; Pearce and Peate 1995). The Th/Yb vs. Ta/Yb plot (Fig. 12c) illustrates that the mafic samples are transitional between E-MORB and OIB, consistent with their REE patterns, suggesting that they formed in an intraplate setting (Fig. 11). Further, on the $\mathrm{TiO}_{2} / \mathrm{Yb}$ vs. $\mathrm{Nb} / \mathrm{Yb}$ diagram, the mafic samples fall into the OIB field partly overlapping with the MORB field indicating potential plume-ridge interaction features (Fig. 12d).

Even though limited in volume, the Middle to Upper Triassic mafic rocks are widespread through the SSZ (e.g., Berberian and King 1981; Badrzadeh et al. 2011; Mousivand et al. 2012) and Central Iran (e.g., Bagheri and Stampfli 2008; Buchs et al. 2013). In the southeastern of SSZ, Arvin et al. (2007) reported calcalkaline, I-type granites with a Sm-Nd 'errorchron' age of $199 \pm 30 \mathrm{Ma}$ and interpreted this suite to have formed in a volcanic arc setting due to onset of Neotethys subduction. However, the analytical error is large, and is within error similar Middle Jurassic calc-alkaline granitoids and gabbros (Shakerardakani et al. 2015; Hassanzadeh and Wernicke 2016). Mousivand et al. (2012) found arc tholeiites with a U-Pb zircon age of $191 \pm 12 \mathrm{Ma}$ (Early Jurassic) associated with massive sulphide deposits (see also Badrzadeh et al. 2011) also interpreted to relate to Neotethys subduction. Consequently, the Upper Triassic mafic rocks of the study area were formed during the stage of the Neotethys oceanic expansion prior to the Early Jurassic onset of subduction.

Berberian and King (1981) suggested that after the quiet Paleozoic Gondwanan tectono-sedimentary regime in Iran, 

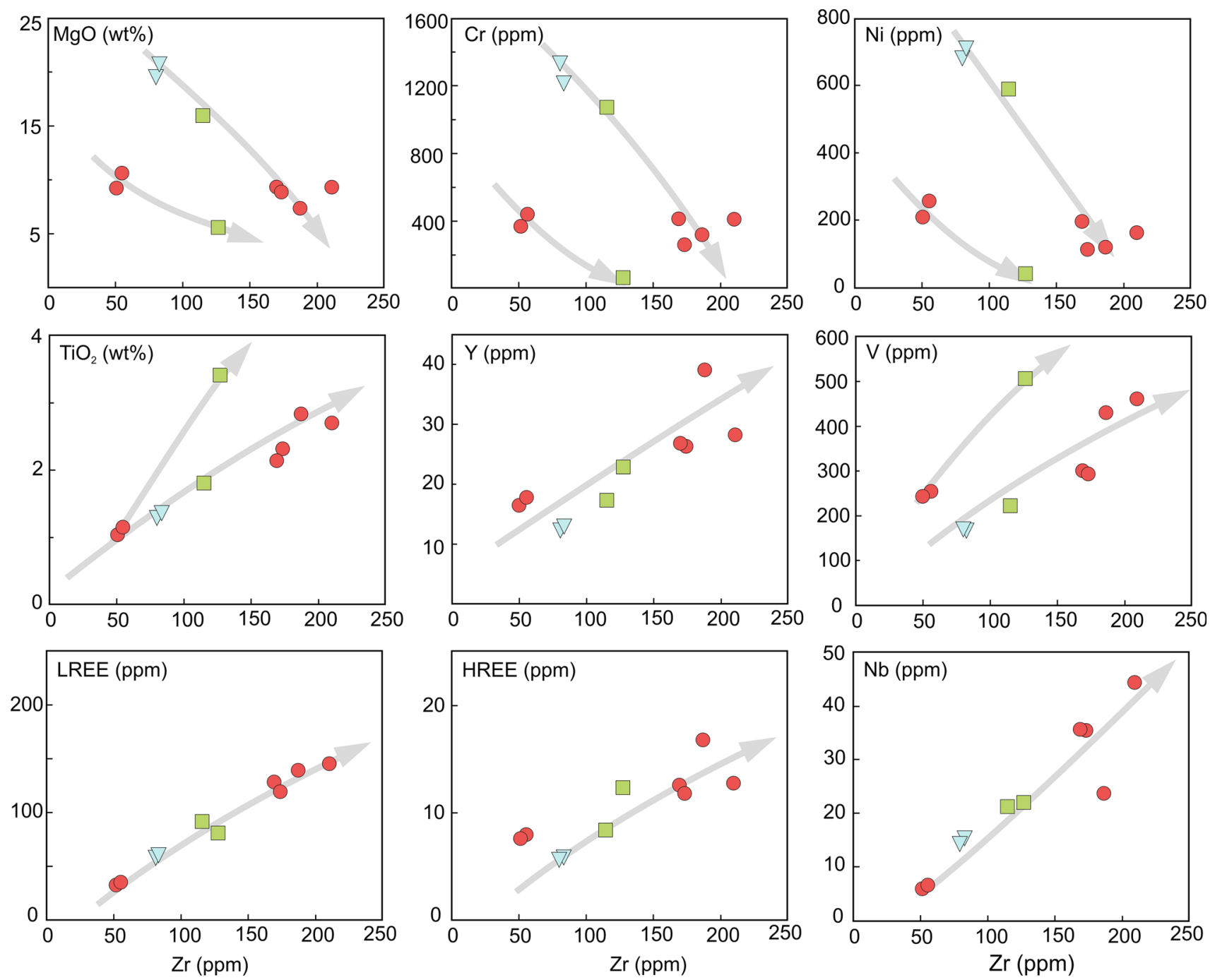

Fig. 9 Variation in selected major and trace elements vs. Zr for various mafic rocks from the June Complex. Arrows show inferred fractionation trends of mafic rocks

tectonic activity began by continental rifting and alkali volcanism in the late Paleozoic-early Mesozoic and was followed by sea-floor spreading preserved in the Upper Triassic ophiolitic remnants exposed along the Main Zagros reverse fault (e.g., Saccani et al. 2013a).

\subsection{Tectonic model}

The continental crust of the SSZ has been thinned during Permian and Triassic times and the pressure estimates of clinopyroxene cores might indicate its crystallization in a lower crustal magma chamber (Fig. 13b). From that level, the basaltic magmas has risen into the shallow-water and basinal Triassic sediments. We assume that some magmas were extruded as lavas and pyroclastic rocks and parts were emplaced as sills as testified by the presence of cumulates as testified also by growth of clinopyroxene rims.
The nature of rocks argues that the depositional environment of the Middle-Upper Triassic sedimentary rocks was a shallow marine to basinal shelf facing the Neotethyan Ocean. The thick sedimentary sequence was deposited either in a passive continental margin during a late stage of rifting or rather during the drift stage of the Neotethys Ocean. Recent models assume that the opening of Neotethys started not later than during Middle Triassic times (e.g., Saccani et al. 2014, 2015). The composition of the oceanic crust is influenced by plume-type material (Saccani et al. 2013a, b; Fig. 13a). We argue that the studied mafic rocks represent basalts, which originated from hotspot magmas intruding into the crustal base of the thinned Sanandaj-Sirjan passive margin basement and rose up into shallow levels.

The new data of the central part of SSZ indicates that there are obvious differences to the southernmost part of 

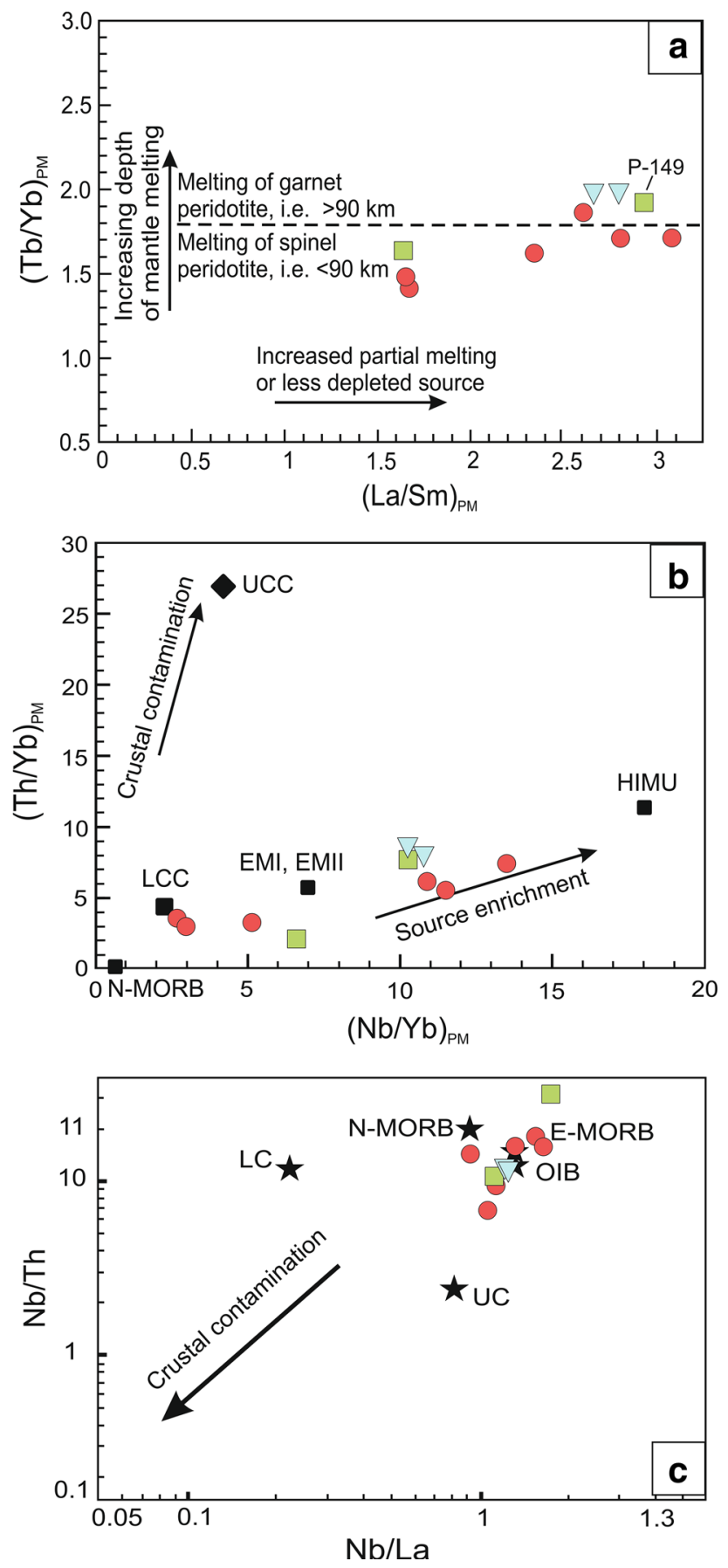

Fig. 10 a $(\mathrm{La} / \mathrm{Sm})_{\mathrm{PM}}$ versus $(\mathrm{Tb} / \mathrm{Yb})_{\mathrm{PM}}$ diagram for the cumulate and isotropic mafic rocks of the June Complex; ratios are normalized to primitive Mantle values of McDonough and Sun (1995). The discrimination dashed line separates two fields for melting of garnet-bearing peridotite and of spinel-bearing peridotite (Wang et al. 2002). b Diagram showing variations in $(\mathrm{Th} / \mathrm{Yb})_{\mathrm{PM}}$ and $(\mathrm{Nb} /$ $\mathrm{Yb})_{\mathrm{PM}}$ ratios. N-MORB composition is from Hofmann (1988), Upper Continental Crust (UCC) composition is from Taylor and McLennan (1985), and enriched mantle (EMI, EMII) and HIMU values are from Condie 2001. $\mathrm{c} \mathrm{Nb/La} \mathrm{vs.} \mathrm{Nb} / \mathrm{Th}$ diagram showing the trend of crustal contamination (after Zhang et al. 2014)

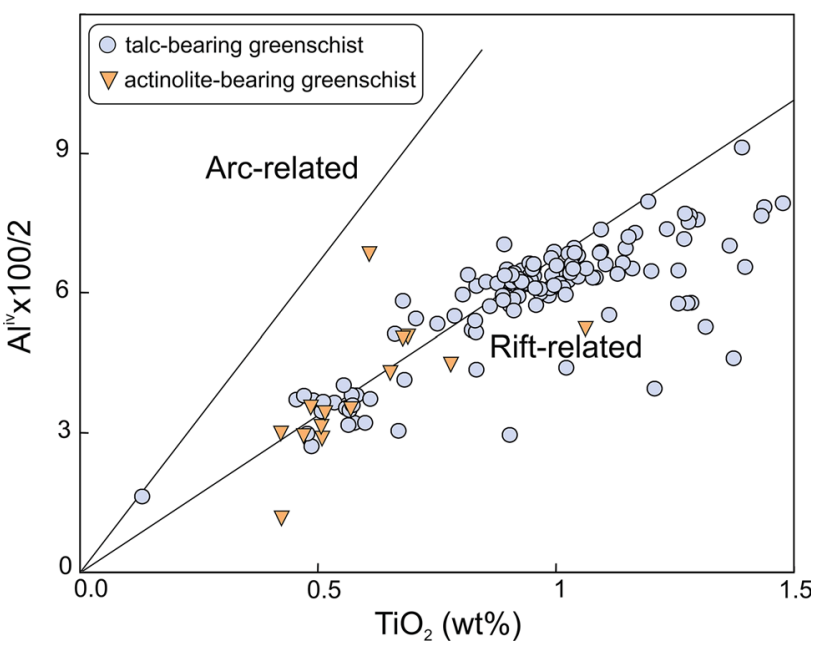

Fig. $11 \mathrm{Alz}\left(=\mathrm{Al}_{\mathrm{iv}} \times 100 / 2\right)$ vs. $\mathrm{TiO}_{2}$ of clinopyroxene composition from the June Complex. The trends of clinopyroxenes in arc- and riftrelated mafic-ultramafic cumulates are from Loucks (1990)

SSZ, where tholeiites (and even boninites) with an early Jurassic age associated with massive sulphide deposits are reported (Mousivand et al. 2012). These potentially indicate a switch from drifting stage to subduction as proposed for the Early Jurassic eclogite metamorphism (Davoudian et al. 2016).

Acknowledgements Open access funding provided by Paris Lodron University of Salzburg. Most of the data represent part of the $\mathrm{PhD}$ thesis of Farzaneh Shakerardakani. F.S. acknowledges support by the Afro-Asiatisches Institut, Salzburg, Austria. We would like to thank Eric Condliffe for performing part of microprobe data. We thank two anonymous reviewers for their careful reviews, which helped to increase the clarity of the presentation of data and interpretation.

Open Access This article is distributed under the terms of the Creative Commons Attribution 4.0 International License (http://crea tivecommons.org/licenses/by/4.0/), which permits unrestricted use, distribution, and reproduction in any medium, provided you give appropriate credit to the original author(s) and the source, provide a link to the Creative Commons license, and indicate if changes were made.

\section{Appendix}

Polished thin sections were analyzed with a fully automated JEOL 8600 electron microprobe at the Department of Geography and Geology, University of Salzburg, Austria. Point analyses were obtained using a $15 \mathrm{kV}$ accelerating voltage and $40 \mathrm{nA}$ beam current. The beam size was set to $5 \mu \mathrm{m}$. Natural and synthetic oxides and silicates were used as standards for major elements. Composition profiles of clinopyroxenes were obtained with a fully automated JEOL JXA8230 microprobe at the School of Earth and 
Fig. 12 a $\mathrm{Y} / \mathrm{Zr}$ plot used for discrimination of magmatic affinity (after MacLean and Barrett 1993). Th/Yb vs. Ta/Yb discrimination diagram (Pearce 1982). Values for N-MORB, E-MORB, and oceanic-island basalt (OIB, within-plate basalt) are from Sun and McDonough (1989). Figures indicate $\mathrm{Zr} / \mathrm{Y}$ ratios. b Discrimination plots of $\mathrm{Nb} / \mathrm{Th}$ vs. $\mathrm{Nb}$ and $\mathbf{c} \mathrm{TiO}_{2} / \mathrm{Yb}$ vs. $\mathrm{Nb} / \mathrm{Yb}$ (after Pearce 2014). Primitive Mantle composition is from Hofmann (1988), Continental Crust, MORB (Mid-Ocean Ridge Basalts), OIB (Ocean-Island Basalt) and Arc Volcanics from Schmidberger and Hegner (1999)
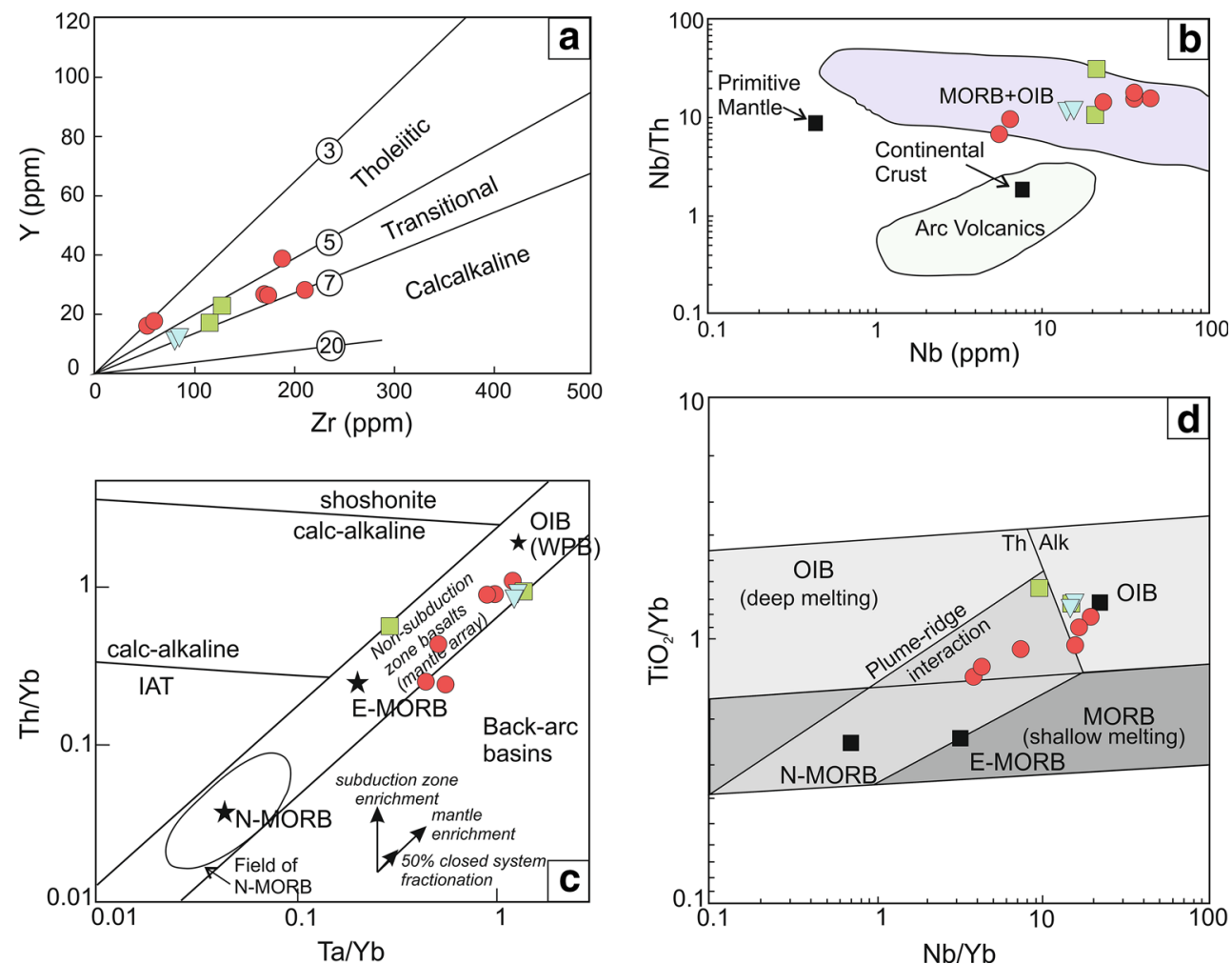

SSE

Exhumed sub-continental mantle

Seamount

\section{E- and P-MORBs Oceanic mantle}

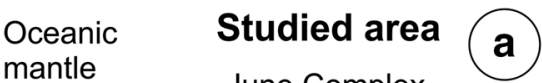

NNW

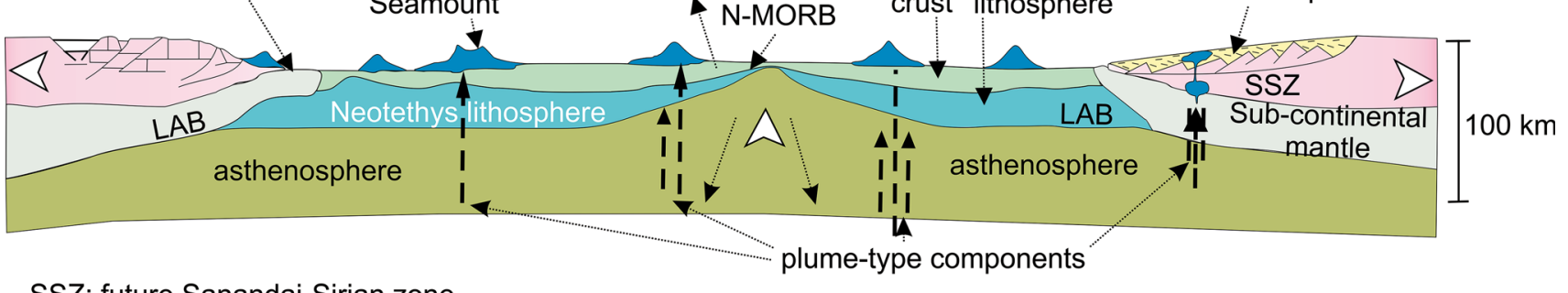

SSZ: future Sanandaj-Sirjan zone

LAB: lithospheric-asthenospheric boundary (b)

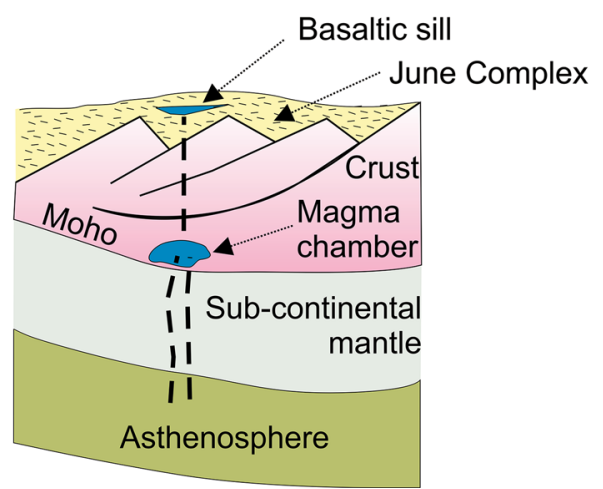

Fig. 13 a Tectonic model of the emplacement of the mafic rocks in the June Complex. The model is largely modified after Saccani et al. (2013a), who assumed no plume-MORB interaction in the northeastern/northern side of the Neotethyan Ocean. b Detail of the suggested emplacement of basalts in the June Complex rising up through the Sanandaj-Sirjan crust 
Environment, University of Leeds, using a $15 \mathrm{kV}$ accelerating voltage and $15 \mathrm{nA}$ beam current, with measurements made at $\sim 5 \mu \mathrm{m}$ and $\sim 10 \mu \mathrm{m}$. Structural formulas for all amphiboles were calculated according to Holland and Blundy (1994). We used the Mathematica packagebased software PET (Dachs 2004) for mineral formula calculation (including nomenclature for amphibole according to the IMA scheme), calculation and plotting of mineral compositions.

Whole-rock major and trace elements were determined on fresh chips of whole rock samples powdered in an agate mill and preserved in a desiccator after being dried in an oven at $105{ }^{\circ} \mathrm{C}$ for $2 \mathrm{~h}$. Major and trace element compositions were analysed by means of XRF (Rigaku RIX 2100) and ICP-MS (PE 6100 DRC), respectively, at the State Key Laboratory of Continental Dynamics, Northwest University, China. For major element analysis, $0.5 \mathrm{~g}$ sample powders were mixed with $3.6 \mathrm{~g} \mathrm{Li}_{2} \mathrm{~B}_{4} \mathrm{O}_{7}, 0.4 \mathrm{~g} \mathrm{LiF}, 0.3 \mathrm{~g}$ $\mathrm{NH}_{4} \mathrm{NO}_{3}$ and minor $\mathrm{LiBr}$ in a platinum pot, and melted to be a glass disc in a high frequency melting instrument prior to analysis. For trace element analysis, sample powders were digested using $\mathrm{HF}+\mathrm{HNO}_{3}$ mixture in a high-pressure Teflon bombs at $190{ }^{\circ} \mathrm{C}$ for $48 \mathrm{~h}$. Analyses of USGS and Chinese national rock standards (BCR-2, GSR-1 and GSR-3) indicate that analytical precision and accuracy for major elements are both generally better than $5 \%$, and for most of the trace elements except for transition metals better than 2 and $10 \%$, respectively. Additionally, one sample (P-136) was randomly selected to be analysed twice to test the accuracy.

Sample P-147, an actinolite-bearing greenschist, was selected for $\mathrm{Sr}$ and $\mathrm{Nd}$ isotope geochemistry (for comparison with other mafic rocks of the region, Shakerardakani et al. 2017). Details of analytical techniques, accuracy and precision can be found in von Quadt et al. (1999). For the isotope analyses, $\sim 50 \mathrm{mg}$ of whole-rock powder was dissolved in $\mathrm{HF}$ and $\mathrm{HNO}_{3}$, followed by $\mathrm{Nd}$ and $\mathrm{Sr}$ separation by exchange chromatography techniques on $100 \mu \mathrm{l}$ TEP columns with Sr Tru and Ln spec Eichrom resin. Nd was purified on a Ln spec column ( $2 \mathrm{ml})$ using $0.22 \mathrm{n}$ and $0.45 \mathrm{n} \mathrm{HCl}$. Nd and $\mathrm{Sr}$ isotopes were analysed on a ThermoPlus mass spectrometer by static mode measurements at ETH Zürich. Sr was loaded with a Ta emitter on Re filaments. The measured ${ }^{87} \mathrm{Sr} /{ }^{86} \mathrm{Sr}$ ratio was normalized to an ${ }^{88} \mathrm{Sr} /{ }^{86} \mathrm{Sr}$ value of 8.37521 . The mean ${ }^{87} \mathrm{Sr} /{ }^{86} \mathrm{Sr}$ value of the NBS 987 standard obtained during the period of measurements was $0.710252 \pm 12(2 \sigma, \mathrm{n}=18)$. A mass fractionation correction of $1.1 \%$ per atomic mass unit was applied, based on replicate analyses of the NBS 982 reference material. $\mathrm{Nd}$ was loaded with $2 \mathrm{n} \mathrm{HCl}$ on Re filaments. The measured ${ }^{143} \mathrm{Nd} /{ }^{144} \mathrm{Nd}$ ratio was normalized to ${ }^{146} \mathrm{Nd} /{ }^{144} \mathrm{Nd}$ value of 0.7219 . The mean ${ }^{143} \mathrm{Nd} /{ }^{144} \mathrm{Nd}$ value of the Nd Merck standard obtained during the period of measurements was $0.511730 \pm 1(2 \sigma, \mathrm{n}=12)$. Age-corrected $\mathrm{Nd}$ and $\mathrm{Sr}$ isotope ratio was calculated using $\mathrm{Rb}, \mathrm{Sr}$, $\mathrm{Sm}$ and Nd concentrations determined by LA-ICP-MS.

\section{References}

Agard, P., Omrani, J., Jolivet, L., \& Mouthereau, F. (2005). Convergence history across Zagros (Iran): Constraints from collisional and earlier deformation. International Journal of Earth Sciences, 94, 401-419.

Agard, P., Omrani, J., Jolivet, L., Whitchurch, H., Vrielynck, B., Spakman, W., et al. (2011). Zagros orogeny: A subductiondominated process. Geological Magazine, 148, 692-725.

Alavi, M. (1994). Tectonics of the Zagros Orogenic belt of Iran: New data and interpretations. Tectonophysics, 229, 211-238.

Arvin, M., Pan, Y., Dargahi, S., Malekizadeh, A., \& Babaei, A. (2007). Petrochemistry of the Siah-Kuh granitoid stock southwest of Kerman, Iran: Implications for initiation of Neo-Tethys subduction. Journal of Asian Earth Sciences, 30, 474-489.

Badrzadeh, Z., Barrett, T. J., Peter, J. M., Gimeno, D., Sabzehei, M., \& Aghazadeh, M. (2011). Geology, mineralogy and sulfur isotope geochemistry of the Sargaz $\mathrm{Cu}-\mathrm{Zn}$ volcanogenic massive sulfide deposit, Sanandaj-Sirjan zone, Iran. Mineralium Deposita, 46, 905-923.

Bagheri, S., \& Stampfli, G. M. (2008). The Anarak, Jandaq and Poshte-Badam metamorphic complexes in central Iran: New geological data, relationships and tectonic implications. Tectonophysics, 451, 123-155.

Baker, J. A., Menzies, M. A., Thirlwall, M. F., \& Macpherson, C. G. (1997). Petrogenesis of Quaternary intraplate volcanism, Sana'a, Yemen: Implications for plume-lithosphere interaction and polybaric melt hybridization. Journal of Petrology, 38, 1359-1390.

Berberian, M., \& King, G. C. P. (1981). Towards a paleogeography and tectonic evolution of Iran. Canadian Journal of Earth Sciences, 18, 210-265.

Besse, J., Torcq, F., Gallet, Y. L., Ricou, E., Krystyn, L., \& Saidi, A. (1998). Late Permian to Late Triassic palaeomagnetic data from Iran: Constraints on the migration of the Iranian block through the Tethyan Ocean and initial destruction of Pangaea. Geophysical Journal International, 135(1), 77-92.

Blundy, J. D., \& Wood, B. J. (1991). Crystal-chemical controls on the portioning of $\mathrm{Sr}$ and $\mathrm{Ba}$ between plagioclase feldspar, silicate melts, and hydrothermal solutions. Geochimica et Cosmochimica Acta, 55, 193-209.

Boynton, W. V. (1984). Cosmochemistry of the rare earth elements: Meteorite studies. In P. Henderson (Ed.), Rare earth element geochemistry (pp. 63-114). Amsterdam: Elsevier.

Buchs, D. M., Bagheri, S., Martin, L., Hermann, J., \& Arculus, R. (2013). Paleozoic to Triassic ocean opening and closure preserved in Central Iran: Constraints from the geochemistry of meta-igneous rocks of the Anarak area. Lithos, 172-173, 267-287.

Condie, K. C. (2001). Mantle plumes and their record in Earth History. Oxford: Cambridge University Press.

Dachs, E. (2004). PET: Petrological elementary tools for mathematica (R): An update. Computers \& Geosciences, 30, 173-182.

Davoudian, A.R., Genser, J., Neubauer, F., Shabanian, N. (2011). The new findings of the time of Neotethys subduction on the base of ${ }^{40} \mathrm{Ar}-{ }^{39} \mathrm{Ar}$ age dating eclogite samples from north of Shahrekord. In 15th symposium of Geological Society of Iran. 
Davoudian, A. R., Genser, J., Neubauer, F., \& Shabanian, N. (2016). ${ }^{40} \mathrm{Ar}-{ }^{39} \mathrm{Ar}$ mineral ages of eclogite from North Shahrekord in the Sanandaj-Sirjan Zone, Iran: Implications for the tectonic evolution of Zagros orogen. Gondwana Research, 37, 216-240.

Deng, H., Kusky, T., Polat, A., Wang, L., Wang, J., \& Wang, S. (2013). Geochemistry of Neoarchean mafic volcanic rocks and late mafic dikys in the Zanhung Complex, Central Orogenic Belt, North China Craton: Implications for geodynamic setting. Lithos, 175-176, 193-212.

Farahat, E. S. (2011). Geotectonic significance of Neoproterozoic amphibolites from the Central Eastern Desert of Egypt: A possible dismembered sub-ophiolitic metamorphic sole. Lithos, 125, 781-794.

Fergusson, C. L., Nutman, A. P., Mohajjel, M., \& Bennett, V. (2016). The Sanandaj-Sirjan zone in the Neo-Tethyan suture, western Iran: Zircon U-Pb evidence of late Palaeozoic rifting of northern Gondwana and mid-Jurassic orogenesis. Gondwana Research, 40, 43-57.

Ghasemi, A., \& Talbot, C. J. (2006). A new tectonic scenario for the Sanandaj-Sirjan Zone (Iran). Journal of Asian Earth Sciences, 26, 683-693.

Gidon, M., Berthier, F., Billiault, J. P., Halbronn, B., \& Maurizot, P. (1974). Sur les caractères et l'ampleur du coulissement de la "Main Fault" dans la region de Borudjerd-Dorud (Zagros oriental, Iran). Comptes Rendus de l'Académie des Sciences, 278, 701-704.

Green, T. H. (1967). An experimental investigation of sub-solidus assemblages formed at high pressure in high alumina basalt, Kyanite eclogite and grosspydite compositions. Contributions to Mineralogy and Petrology, 16, 84-114.

Hassanzadeh, J., \& Wernicke, B. P. (2016). The Neotethyan Sanandaj-Sirjan zone of Iran as an archetype for passive margin-arc transitions. Tectonics, 35, 586-621.

Herzberg, C. (1995). Generation of plume magmas through time-An experimental perspective. Chemical Geology, 126, 1-16.

Himmelberg, G. R., \& Loney, R. A. (1995). Characteristics and petrogenesis of Alaskan-type ultramafic-mafic intrusions, southeastern Alaska. U.S. Geological Survey Professional Paper, 1564.

Hofmann, A. W. (1988). Chemical differentiation of the Earth: The relationship between mantle, continental crust and oceanic crust. Earth and Planetary Science Letters, 90, 297-314.

Holland, T., \& Blundy, J. (1994). Nonideal interactions in calcic amphiboles and their bearing on amphibole-plagioclase thermometry. Contributions to Mineralogy and Petrology, 116, 433-447.

Irvine, T. N., \& Baragar, W. R. A. (1972). A guide to the chemical classification of the common volcanic rocks. Canadian Journal of Earth Science, 8, 523-548.

Kempton, P., \& Harmon, R. (1992). Oxygen isotope evidence for large-scale hybridization of the lower crust during magmatic underplating. Geochimica et Cosmochimica Acta, 56, 971-986.

Kerrich, R., \& Xie, Q. (2002). Compositional recycling structure of an Archaean superplume: Nb-Th-U-LREE systematics of Archaean komatiites and basalts revisited. Contributions to Mineralogy and Petrology, 142, 476-484.

Khudoley, A. K., Prokopiev, A. V., Chamberlain, K. R., Ernst, R. E., Jowitt, S. M., Malyshev, S. V., et al. (2013). Early Paleozoic mafic magmatic events on the eastern margin of the Siberian craton. Lithos, 174, 44-56.

Kushiro, I. (1969). Clinopyroxene solid solutions formed by reactions between diopside and plagioclase at high pressures. Mineralogical Society of America, Special Paper, 2, 179-191.

Le Maitre, R. W., Bateman, P., Dudek, A., Keller, J., Lameyre, J., Le Bas, M. J., et al. (1989). A classification of igneous rocks and glossary of terms: Recommendations of the International Union of Geological Sciences subcommission on the systematics of igneous rocks. Oxford: Blackwell Scientific Publications.

Leake, B. E., Woolley, A. R., Arps, C. E. S., Birch, W. D., Gilbert, M. C., Grice, J. D., et al. (1997). Nomenclature of amphiboles: Report of the subcommittee on amphiboles of the International Mineralogical Association, commission on new minerals and mineral names. American Mineralogist, 82, 1019-1037.

Loucks, R. B. (1990). Discrimination of ophiolitic from nonophiolitic ultramafic-mafic allochthons in orogenic belts by the Al/Ti ratio in Clinopyroxene. Geology, 18, 346-349.

MacLean, W. H., \& Barrett, T. J. (1993). Lithogeochemical techniques using immobile elements. Journal of Geochemical Exploration, 48, 109-133.

McDonough, W. F., \& Sun, S. S. (1995). The composition of the Earth. Chemical Geology, 120, 223-254.

Miyashiro, A. (1978). Nature of alkali volcanic rock series. Contributions to Mineralogy and Petrology, 66, 91-104.

Mohajjel, M., \& Fergusson, C. (2000). Dextral transpression in Late Cretaceous continental collision Sanandaj-Sirjan zone western Iran. Journal of Structural Geology, 22, 1125-1139.

Mohajjel, M., \& Fergusson, C. (2014). Jurassic to Cenozoic tectonics of the Zagros Orogen in northwestern Iran. International Geology Review, 56, 263-287.

Mohajjel, M., Fergusson, C., \& Sahandi, M. R. (2003). CretaceousTertiary convergence and continental collision, Sanandaj-Sirjan Zone, western Iran. Journal of Asian Earth Sciences, 21, 397-412.

Morimoto, N. (1989). Nomenclature of pyroxenes. Canadian Mineralogist, 27, 143-156.

Mousivand, F., Rastad, E., Meffre, S., Peter, J. M., Mohajjel, M., Zaw, K., et al. (2012). Age and tectonic setting of the Bavanat $\mathrm{Cu}-\mathrm{Zn}$-Ag Besshi-type volcanogenic massive sulfide deposit, southern Iran. Mineralium Deposita, 47, 911-931.

Nimis, P., \& Taylor, W. R. (2000). Single clinopyroxene thermobarometry for garnet peridotites. Part I. Calibration and testing of a Cr-in-Cpx barometer and an enstatite-in-Cpx thermometer. Contributions to Mineralogy and Petrology, 139, 541-554.

Nutman, A. P., Mohajjel, M., Bennett, V. C., \& Fergusson, C. L. (2014). Gondwanan Eoarchean-Neoproterozoic ancient crustal material in Iran and Turkey: Zircon $\mathrm{U}-\mathrm{Pb}-\mathrm{Hf}$ isotopic evidence. Canadian Journal of Earth Sciences, 51, 272-285.

Ordóñez-Calderón, J. C., Polat, A., Fryer, B. J., \& Gagnon, J. E. (2011). Field and geochemical characteristics of Mesoarchean to Neoarchean volcanic rocks in the Storø greenstone belt, SW Greenland: Evidence for accretion of intra-oceanic volcanic arcs. Precambrian Research, 184, 24-42.

Pearce, J. A. (1982). Trace element characteristics of lavas from destructive plate boundaries. In E. S. Thorpe (Ed.), Andesites (pp. 525-548). New York: Wiley.

Pearce, J. A. (1983). Role of sub-continental lithosphere in magma genesis at active continental margins. In C. J. Hawkesworth \& M. J. Norry (Eds.), Continental basalts and mantle xenoliths (pp. 230-249). Nantwich: Shiva.

Pearce, J. (2014). Immobile element fingerprinting of ophiolites. Elements, 10(2), 101-108.

Pearce, J. A., \& Norry, M. J. (1979). Petrogenetic implications of Ti, $\mathrm{Zr}, \mathrm{Y}$, and $\mathrm{Nb}$ variations in volcanic rocks. Contributions to Mineralogy and Petrology, 69, 33-47.

Pearce, J. A., \& Peate, D. W. (1995). Tectonic implications of the composition of volcanic arc magmas. Annual Review of Earth and Planetary Sciences, 23, 251.

Perchuck, L. L., Aranovich, L. Ya., Podlesskii, K. K., Lavarant'eva, I. V., Gerasimov, V. Y., Fed'kin, V. V., et al. (1985). Precambrian granulites of the Aldan Shield, Eastern Siberia, USSR. Journal of Metamorphic Geology, 3(3), 265-310. 
Polat, A., \& Hofmann, A. W. (2003). Alteration and geochemical patterns in the 3.7-3.8 Ga Isua greenstone belt, West Greenland. Precambrian Research, 126, 197-218.

Prokopiev, A. V., Khudoley, A. K., Koroleva, O. V., Kazakova, G. G., Lokhov, D. K., Malyshev, S. V., et al. (2016). The Early Cambrian bimodal magmatism in the northeastern Siberian Craton. Russian Geology and Geophysics, 57, 155-175.

Richards, J. P. (2015). Tectonic, magmatic, and metallogenic evolution of the Tethyan orogeny: From subduction to collision. Ore Geology Reviews, 70, 323-345.

Saccani, E., Allahyari, K., Beccaluva, L., \& Bianchini, G. (2013a). Geochemistry and petrology of the Kermanshah ophiolites (Iran): Implication for the interaction between passive rifting, oceanic accretion, and OIB-type components in the Southern Neo-Tethys Ocean. Gondwana Research, 24, 392-411.

Saccani, E., Allahyari, K., \& Rahimzadeh, B. (2014). Petrology and geochemistry of mafic magmatic rocks from the Sarve-Abad ophiolites (Kurdistan region, Iran): Evidence for interaction between MORB-type asthenosphere and OIB-type components in the southern Neo-Tethys Ocean. Tectonophysics, 621, 132-147.

Saccani, E., Azimzadeh, Z., Dilek, Y., \& Jahangiri, A. (2013b). Geochronology and petrology of the Early Carboniferous Misho Mafic Complex (NW Iran), and implications for the melt evolution of Paleo-Tethyan rifting in Western Cimmeria. Lithos, 162-163, 264-278.

Saccani, E., Delavari, M., Beccaluva, L., \& Amini, S. (2010). Petrological and geochemical constraints on the origin of the Nehbandan ophiolitic complex (eastern Iran): Implication for the evolution of the Sistan Ocean. Lithos, 117, 209-228.

Saccani, E., Dilek, Y., Marroni, M., \& Pandolfi, L. (2015). Continental margin ophiolites of Neotethys: Remnants of ancient Ocean-Continent Transition Zone (OCTZ) Lithosphere and their geochemistry, mantle sources and melt evolution patterns. Episodes Journal of International Earth Sciences, 38, 230-249.

Sahandi, M. R., Radfar, J., Hoseinidoust, J., \& Mohajjel, M. (2006). Explanatory text of the Shazand Quadrangle Map. Tehran: Geological Survey of Iran.

Sappin, A. A., Constantin, M., \& Clark, T. (2012). Petrology of mafic and ultramafic intrusions from the Portneuf-Mauricie Domain, Grenville Province, Canada: Implications for plutonic complexes in a Proterozoic island arc. Lithos, 154, 277-295.

Schmidberger, S. S., \& Hegner, E. (1999). Geochemistry and isotope systematics of calcalkaline volcanic rocks from the Saar-Nahe basin (SW German)-implications for Late Variscan orogenic development. Contributions to Mineralogy and Petrology, 135, 373-385.

Şengör, A. M. C. (1990). A new model for the late PaleozoicMesozoic tectonic evolution of Iran and implications for Oman. In A. H. F. Robertson, M. P. Searle, \& A. C. Reis (Eds.), The geology and tectonics of the Oman region (Vol. 49, pp. 797-831). London: Geological Society Special Publication.

Shakerardakani, F., Neubauer, F., Bernroider, M., Von Quadt, A., Liu, X., Genser, J., et al. (2017). Geochemical and isotopic evidence for Carboniferous rifting-mafic dykes in the central SanandajSirjan zone (Dorud-Azna, West Iran). Geologica Carpathica, 68(3), 229-247.

Shakerardakani, F., Neubauer, F., Masoudi, F., Mehrabi, B., Liu, X., Dong, Y., et al. (2015). Panafrican basement and Mesozoic gabbro in the Zagros orogenic belt in the Dorud-Azna region
(NW Iran): Laser-ablation ICP-MS zircon ages and geochemistry. Tectonophysics, 647-648, 146-171.

Stampfli, G. M., \& Borel, G. D. (2002). A plate tectonic model for the Paleozoic and Mesozoic constrained by dynamic plate boundaries and restored synthetic oceanic isochrons. Earth and Planetary Science Letters, 196, 17-33.

Stampfli, G. M., Borel, G. D., Cavazza, W., Mosar, J., \& Ziegler, P. A. (2001). Palaeotectonic and palaeogeographic evolution of the western Tethys and PeriTethyan domain (IGCP Project 369). Episodes, 24, 222-228.

Stampfli, G. M., Marcoux, J., \& Baud, A. (1991). Tethyan margins in space and time. Palaeogeography, Palaeoclimatology, Palaeoecology, 87, 373-409.

Sun, S. S., \& McDonough, W. F. (1989). Chemical and isotopic systematic of ocean basalts: Implication for mantle composition and processes. In A. D. Saunders \& M. J. Norry (Eds.), Magmatism in the ocean basins (Vol. 42, pp. 313-345). London: Geological Society [London] Special Publication.

Taylor, S. R., \& McLennan, S. M. (1985). The continental crust: Its composition and evolution: An examination of the geochemical record preserved in sedimentary rocks. Oxford: Blackwell Scientific.

Viccaro, M., \& Cristofolini, R. (2008). Nature of mantle heterogeneity and its role in the short-term geochemical and volcanological evolution of Mt. Etna (Italy). Lithos, 105, 272-288.

Von Quadt, A., Gunther, D., Frischknecht, R., \& Dietrich, V. (1999). Minor and trace element determinations in Li2B407 fused USGS standard materials calibrated without matrix-matched standards using laser ablation ICP-MS. Journal of Conference Abstracts, 4, 819.

Wang, K., Plank, T., Walker, J. D., \& Smith, E. I. (2002). A mantle melting profile across the basin and range, SW USA. Journal of Geophysical Research, 107(B1), 2017.

Winchester, J. A., \& Floyd, P. A. (1976). Geochemical magma type discrimination application to altered and metamorphosed basic igneous rocks. Earth and Planetary Science Letters, 28, 459-469.

Winchester, J. A., \& Floyd, P. A. (1977). Geochemical discrimination of different magma series and their differentiation products using immobile elements. Chemical Geology, 20, 325-343.

Woodland, A. B. (2009). Ferric iron contents of clinopyroxene from cratonic mantle and partitioning behavior with garnet. Lithos, $112,1143-1149$.

Xia, L. Q., Xu, X. Y., Xia, Z. C., Li, X. M., Ma, Z. P., \& Wang, L. S. (2004). Petrogenesis of Carboniferous rift-related volcanic rocks in the Tianshan, northwestern China. Geological Society of America Bulletin, 116, 419-433.

Zanchi, A., Malaspina, N., Zanchetta, S., Berra, F., Benciolini, L., Bergomi, M., et al. (2015). The Cimmerian accretionary wedge of Anarak, Central Iran. Journal of Asian Earth Sciences, 102, $45-72$.

Zhang, C. L., Zou, H. B., Yao, C. Y., \& Dong, Y. G. (2014). Origin of Permian gabbroic intrusions in the southern margin of the Altai Orogenic belt: A possible link to the Permian Tarim mantle plume? Lithos, 204, 112-124.

Zhu, Y., \& Ogasawara, Y. (2004). Clinopyroxene phenocrysts (with green salite cores) in trachybasalts: Implications for two magma chambers under the Kokchetav UHP massif, North Kazakhstan. Journal of Asian Earth Sciences, 22, 517-527. 